\title{
Immunopathogenesis of pancreatitis
}

\author{
T Watanabe ${ }^{1,2}, \mathrm{M} \mathrm{Kudo}^{1}$ and W Strober ${ }^{2}$
}

The conventional view of the pathogenesis of acute and chronic pancreatitis is that it is due to a genetic- or environmentbased abnormality of intracellular acinar trypsinogen activation and thus to the induction of acinar cell injury that, in turn, sets in motion an intra-pancreatic inflammatory process. More recent studies, reviewed here, present strong evidence that while such trypsinogen activation is likely a necessary first step in the inflammatory cascade underlying pancreatitis, sustained pancreatic inflammation is dependent on damage-associated molecular patterns-mediated cytokine activation causing the translocation of commensal (gut) organisms into the circulation and their induction of innate immune responses in acinar cells. Quite unexpectedly, these recent studies reveal that the innate responses involve activation of responses by an innate factor, nucleotide-binding oligomerization domain 1 (NOD1), and that such NOD1 responses have a critical role in the activation/production of nuclear factor-kappa B and type I interferon. In addition, they reveal that chronic inflammation and its accompanying fibrosis are dependent on the generation of IL-33 by injured acinar cells and its downstream induction of Tcells producing IL-13. These recent studies thus establish that pancreatitis is quite a unique form of inflammation and one susceptible to newer, more innovative therapy.

\section{INTRODUCTION}

Pancreatitis is a major inflammatory disease of the gastrointestinal tract and one that has so far been resistant to specific treatment. ${ }^{1-4}$ It manifests as an acute or chronic disease, each displaying some overlapping and some unique characteristics: the acute form is a new-onset inflammation occurring in a previously uninflamed pancreas that may resolve before causing permanent damage to the pancreas; in contrast, the chronic form is an ongoing pancreatic inflammation in which the underlying inflammation similar to that driving the acute disease is accompanied by processes leading to pancreatic atrophy and fibrosis. ${ }^{2,5}$

Over the past two decades, a great deal has been learned concerning its pathogenesis, particularly the role of inappropriate trypsinogen activation in the initiation of the inflammatory process. This has been well-discussed in several current reviews and therefore will be only summarized here. ${ }^{6,7}$ Until recently, however, the relation of pancreatitis to the gut microbiome and to related factors that drive the pathological immunological response has been shrouded in mystery. In this review, we will focus on this aspect of pancreatitis pathogenesis and try to establish the thesis that innate immune responses are an integral part of the pathogenesis of pancreatitis.

\section{TRYPSINOGEN ACTIVATION AND PANCREATITIS}

The most widely held understanding of the pathogenesis of pancreatitis is that it is an inflammation initiated and/or sustained by a disturbance in pancreatic acinar cell control of pancreatic digestive enzymes. ${ }^{1-4,6-8}$ To understand how this may be the case it is important to know that digestive enzymes synthesized in pancreatic acinar cells are normally maintained in an inactive form (i.e., as zymogens such as trypsinogen) while in the acinar cells and in the draining ducts and are converted to an active form (such as trypsin) only upon entry into the gut lumen by enterokinase or trypsin itself. ${ }^{6-9}$ Thus, factors that cause premature intra-acinar cell activation of the inactive enzymes have the potential of causing auto-digestion of acinar cells and ensuing events that cause sustained pancreatic inflammation. Several mechanisms of such premature and inappropriate activation of normally inactive zymogens have been put forward and include trypsinogen autoactivation, activation of trypsinogen by lysosomal hydrolase cathepsin B (CatB) caused by shifting of trypsinogen into cellular compartments rich in proteases (lysosomes) and imbalances between degrading and activating acinar cell cathepsins. It should also be noted that activation of pancreatic proteases requires the presence of

${ }^{1}$ Department of Gastroenterology and Hepatology, Kindai University Faculty of Medicine, Osaka-Sayama, Osaka, Japan and ${ }^{2}$ Mucosal Immunity Section, Laboratory of Host Defenses, National Institute of Allergy and Infectious Diseases, National Institutes of Health, Bethesda, Maryland, USA. Correspondence: T Watanabe or W Strober (tomohiro@med.kindai.ac.jp or wstrober@niaid.nih.gov) 
increased intracellular calcium concentrations; thus, disturbances in such concentrations could be a contributing cause of pancreatitis. ${ }^{6-9}$

This "enzyme (trypsin) activation-centered" view of the pathogenesis of pancreatitis derives strong support from studies of genetic defects in humans that pre-dispose individuals to the development of pancreatitis. ${ }^{10}$ For example, various mutations in the gene encoding cationic trypsinogen, the main form of trypsinogen subject to activation after secretion (the protease serine 1 (PRSS1) gene) cause an autosomal dominant susceptibility to acute and chronic pancreatitis because these mutations render the molecule more susceptible to activation or less able to undergo intracellular autolysis. ${ }^{10}$ Similarly, mutations in the gene encoding an inhibitor of activated trypsin that is normally present within the acinar cell (the serine protease inhibitor, Kazal type I (SPINK1) gene) that renders the inhibitor incapable of inhibition cause an autosomal recessive susceptibility to pancreatitis. ${ }^{10}$

Additional support for the concept that inappropriate trypsinogen activation initiates pancreatitis comes from studies of experimental models of pancreatitis. The most widely studied of such models is the pancreatitis induced in mice by administration of "supra-maximal" amounts of the pancreatic secretagogue, cholecystokinin (CCK) or, more commonly, by administration of the CCK analog, cerulein. The reason why pancreatitis is caused by high doses of CCK/cerulein (and not physiological doses) is somewhat unclear. However, there is evidence that high-dose CCK/cerulein stimulation causes activation of acinar cells via low-affinity CCK receptors rather than high-affinity receptors and this form of activation has the effect of causing intracellular ultra-structural changes that result in inhibition of acinar cell secretion. ${ }^{8}$ This, in turn, results in the rapid accumulation of trypsinogen within acinar cells and exposure of accumulated trypsinogen to factors that result in trypsinogen activation. ${ }^{8}$ As indicated by the fact that inhibition or deletion of CatB leads to greatly reduced cerulein-induced pancreatitis, one possible trypsinogen activating factor is the co-localization of zymogen granules with an activating CatB. ${ }^{8}$

A challenge to the concept that trypsinogen activation within acinar cells is a key initiating event in cerulein-induced pancreatitis (i.e., in pancreatitis in general) comes from recent studies showing that mice in whom there is deletion in T7 isoform of trypsinogen (the form of trypsinogen equivalent to "cationic trypsinogen" that has been implicated in acinar cell destruction and pancreatitis in humans) exhibit absence of trypsinogen or chymotrypsin activation and a $50 \%$ reduction in acinar cell necrosis following initiation of cerulein-induced pancreatitis. Nevertheless, these mice exhibited similar levels of pancreatitis and lung inflammation as assessed by neutrophil infiltration and pancreatic histology. ${ }^{11}$ Moreover, in additional studies in which mice were given repeated doses of cerulein over many weeks to induce chronic pancreatitis, absence of T7 trypsinogen again had no effect on the development of ceruleininduced chronic pancreatitis. ${ }^{12}$ In both the studies of acute and chronic pancreatitis, T7-deletion was associated with lack of cerulein-induced chymotrypsin and elastase generation so that the effect of T7-deletion was apparently not being compensated by increases in the activation of these zymogens. Finally, whereas both acute and chronic cerulein-induced pancreatitis occurred in mice lacking cationic trypsin, these inflammations were accompanied by the early appearance of nuclear factorkappa B (NF-kB) in acinar cells. This, in turn, implied that induction of a trypsinogen activation-independent inflammatory response was the paramount initiator of pancreatitis.

The overall conclusion drawn from these findings was that whereas early pancreatic injury and inflammation could conceivably occur as a result of trypsinogen activation, both acute and chronic pancreatitis can and does proceed in the absence of such activation. The weakness of this conclusion lies in the fact that deletion of T7 trypsinogen leaves intact the expression of other forms of trypsinogen that can also undergo autoactivation and thus cause pancreatitis in mice if not in humans. ${ }^{13}$ Thus, while in vitro acinar cell necrosis resulting from exposure to super-maximal CCKR stimulation was shown to be dependent on T7 trypsinogen, in vivo acinar cell necrosis induced by cerulein occurred in the absence of T7 trypsinogen, albeit to a reduced extent, presumably due to activation of other trypsinogen isoforms that occurs in vivo. ${ }^{11}$ On this basis, these studies of T7 trypsinogen deletion do not negate the idea that experimental pancreatitis is initiated if not sustained by dysregulation of trypsinogen activation; on the contrary, this idea still holds as does the concept that experimental pancreatitis mimics human pancreatitis where evidence that abnormalities of trypsinogen activation is a primary cause of pancreatitis is buttressed by genetic studies.

A second set of findings raising a question concerning the trypsin-centered concept of pancreatitis development comes from a recent study showing that genetically engineered mice in whom one can induce activation of trypsinogen in acinar cells in the absence of cerulein stimulation do in fact develop acute pancreatitis associated with acinar cell apoptosis and necrosis. ${ }^{14}$ However, in this case trypsinogen activation did not lead to sustained pancreatitis. These findings thus suggest that whereas intracellular trypsinogen activation is dispensable for the development of cerulein-induced pancreatitis as indicated by the T7-deletion studies above, such activation can nevertheless be an initiating cause of pancreatitis. Thus, while they dispute the T7-deletion studies by showing that inappropriate trypsinogen activation can indeed induce acute pancreatitis, they support the T7- deletion studies by showing that other changes in the acinar cells induced by supra-maximal cerulein-mediated CCKR stimulation are necessary to induce a sustained inflammation. ${ }^{15}$

Of particular interest is the relation of trypsinogen activation to the ingestion of alcohol since excess alcohol consumption is one of the leading causes of pancreatitis in humans. ${ }^{5}$ However, it is difficult to induce pancreatitis in experimental animals by administration of alcohol alone and it is thus likely that alcohol is not a primary cause of pancreatitis, but rather a secondary cause that potentiates other pancreatitis inducing factors (at least in rodents). ${ }^{7}$ One possibility in this context is the known ability of alcohol (i.e., ethanol) to cause increased viscosity of pancreatic secretions and thus to favor obstruction 
to pancreatic outflow, a feature of cerulein-induced pancreatitis. The second possibility is that alcohol consumption causes increased gut permeability and the penetration of bacteria and/ or bacterial products into the circulation; as discussed more fully below, this results in increased exposure of acinar cells to gut bacterial components and the activation of the NLRP3 (NOD-like receptor family pyrin domain containing-3) inflammasome due to signaling via toll-like receptor 4 (TLR4). ${ }^{16}$ This idea is fully supported by the clinical observation that plasma concentrations of endotoxin are elevated in patients with alcoholic-related diseases. ${ }^{17}$ The final and perhaps the most important possibility relates to the capacity of ethanol to enhance $\mathrm{CCK}$ activation of $\mathrm{NF}-\kappa \mathrm{B}$ via phosphorylation of protein kinase $\mathrm{C}(\mathrm{PKC}) .{ }^{18}$ This last mechanism suggests that ethanol has a direct role in the stimulation of a key signaling pathway necessary to the induction of pancreatic inflammation.

Cigarette smoking is another risk factor for acute and chronic pancreatitis and one that appears to potentiate the effect of alcohol. ${ }^{19}$ The mechanisms underlying this association are poorly understood and likely to be multifactorial, reflecting the fact that cigarette smoke contains many factors that might affect a number of aspects of pancreatic inflammation.

\section{AUTOPHAGY AND PANCREATITIS}

Autophagy is an essential cellular process through which longlived proteins and cytoplasmic organelles are degraded and used for recycling. ${ }^{20}$ In this process, targeted proteins become entrapped in double-membrane autophagic vacuoles and are then subjected to proteolytic digestion when the autophagic vacuoles are fused with lysosomes. ${ }^{20}$ Given the fact that pancreatitis is marked by acinar cells in which proteolytic enzymes normally sequestered in protective granules are released into the cytoplasm it was reasonable to assume that defective autophagic function is an important, even primary, cause of pancreatitis (see reviews by Gukovskaya and Gukovsky). ${ }^{21,22}$ The most compelling evidence in support of this concept comes from studies of mice with specific deletions of key autophagy genes such as that of Diakopoulos et al. who showed that mice with pancreas-specific autophagyrelated 5 (ATG5) deficiency, develop chronic pancreatitis accompanied by acinar cells showing evidence of endoplasmic reticulum stress. ${ }^{23}$ Likewise, Antonucci et al., ${ }^{24}$ showed that mice with pancreatic epithelial cell loss of ATG7, develop chronic pancreatitis and extensive fibrosis, also associated with endoplasmic reticulum stress. Finally, in related studies it was shown by Li et al. ${ }^{25}$ that mice deficient in I $\kappa \mathrm{B}$ kinase $\alpha(\mathrm{IKK} \alpha)$ develop spontaneous chronic pancreatitis and display elevated levels of acinar cell trypsinogen due to defective degradation of proteins in autophagic vesicles. These pathological changes as well as the presence of enhanced oxidative and endoplasmic reticulum stress were thought to be due to accumulation of protein aggregates dependent on $\mathrm{p} 62$, a protein normally cleared by autophagy, since ablation of p62 partially reversed changes due to IKK $\alpha$ deletion. The mechanism of the effect of IKK $\alpha$ deletion on autophagy was somewhat unclear but since
IKK $\alpha$ binds to ATG16L2, defective function of this autophagy protein may be the cause of the defective autophagy. ${ }^{23,25}$

Since deletion of autophagy genes does not occur in human pancreatitis, or even most forms of murine experimental pancreatitis, one must look elsewhere to implicate an autophagy defect in pancreatic inflammation. This could come from autophagic defects arising from abnormalities affecting fusion of lysosomes with autophagosomes or from inhibition of proteolytic degradation of proteins in fused autophagocytic-lysosomal vacuoles. ${ }^{21}$ Relating to the first of these possibilities, Fortunato et al., reported that pancreatitis was accompanied by abnormalities in the function of lysosomal associated membrane protein 2 (LAMP2), a protein necessary for the completion of autophagosome-lysosome fusion. These investigators showed that rats subjected to alcohol and lipopolysaccharide (LPS) exposure display impaired autophagic vacuole-lysosome fusion due to the depletion of LAMP2 in pancreatic acinar cells and thus decreased autophagic protein degradation. ${ }^{26}$ These findings are supported by those of Mareninova et al. who showed more recently that mice deficient in LAMP2 are subject to the spontaneous pancreatitis marked by impaired autophagy. ${ }^{27}$ In addition, they are supported by studies in humans that showed reduced expression of LAMP-2 in pancreatic tissue of patients with pancreatitis.

The second of the above possibilities, namely defective degradation of autophagosomal contents, is also reported to be involved in the development of pancreatitis. ${ }^{28}$ Thus, Mareninova et al. found that autophagosome size and number were greatly increased in pancreatic acinar cells of rats with pancreatitis induced by cerulein or arginine compared with acinar cells of rats subjected to starvation and, furthermore, this was due to greatly decreased protein degradation as compared with starved cells. ${ }^{28}$ In additon, Mareninova et al. found that cathepsin L (CatL) activity, an enzyme that inhibits trypsin activity by degrading both trypsinogen and trypsin, is impaired in mice with pancreatitis whereas cathepsin B (CatB), an enzyme that converts trypsinogen to trypsin is less impaired. Thus, an imbalance between CatL activity and CatB activity was suggested to be one of the mechanisms by which impaired autophagy contributes to pancreatitis.

Despite these various studies of abnormal autophagy in pancreatitis it remains unclear that this process has a primary role in this form of inflammation. The fact that genetic defects of autophagy components caused by gene deletion can lead to pancreatitis in itself may simply reflect that fact that normal pancreatic cell generation of trypsin requires efficient clearance of non-secreted active enzyme by an autophagic mechanism and pancreatitis ensues in the absence of this mechanism. Alternatively, defects in autophagy may be a secondary effect of the pancreatitis. This could be a consequence of a pancreatitisinduced effect on cathepsin enzymes or other intracellular factors that may impair autophagic function. In support of this possibility, Mareninova et al. have shown that CatB causes cleavage of $\mathrm{LAMP}^{27}$ and it is thus possible that generation of CatB as a result of a pancreatitis inducer (see discussion above) 
has an inhibitory effect of autophagic function. It is important to note, however, that even if an autophagy defect is a secondary consequence of pancreatitis it may have a significant ability to aggravate a primary defect since the need to clear increased trypsin generated by the pathological process becomes more critical in the face of a primary defect.

\section{NECROPTOSIS AND PANCREATITIS}

A major feature of pancreatitis, pancreatic acinar cell death, can conceivably occur as a result of apoptosis or necroptosis (programed necrosis). To some extent which of these death processes is operative may be species-dependent since in the rat model of cerulein-induced pancreatitis apoptosis is the dominant cell death process, whereas in the corresponding mouse model, necrosis is the dominant process. ${ }^{29}$ These unexpected species differences are likely related to the fact that the molecular processes underlying apoptosis and necrosis are different but related. Under pathological conditions apoptosis is most often mediated by tumor necrosis factor-alpha (TNF- $\alpha$ ) activation of TNF receptor type 1-associated death domain protein (TRADD)/ Fas-associated protein with death domain (FADD) or receptor-interacting protein kinase 1 (RIP1)/RIP3 followed by activation of caspase- 8 , the actual "executioner" molecule. ${ }^{30}$ In some contrast, necroptosis is mediated by TNF- $\alpha$ activation of RIP1/RIP3 followed by RIP3 binding to and activation of MLKL (mixed lineage kinase domain-like), a newly defined executioner molecule. ${ }^{31}$ Various stimuli that cause the generation of TNF- $\alpha$ or, alternatively, TLR3 or TLR4 signaling can result in necroptosis, but the circumstances under which this mechanism of cell death occurs rather than apoptosis remains to be clarified. Since caspase- 8 inhibits necroptosis, one possibility is that necroptosis occurs when caspase- 8 is not generated or is inactivated; alternatively, it is possible that necroptosis occurs when RIP3 is highly activated, since this kinase is uniquely involved in necroptosis. In line with these possibilities, caspases are activated and RIP is cleaved in rat cerulein pancreatitis whereas an endogenous inhibitor of caspases (XIAP) undergoes degradation in this model. ${ }^{29}$ In contrast, caspase induction in the mouse cerulein model leads to increased apoptosis and decreased necrosis. Thus, caspase and RIP levels (or state of activation) seem to determine whether acinar cell death in cerulein pancreatitis is predominantly mediated by apoptosis or necrosis. ${ }^{29}$

In more recent studies of acinar cell death in cerulein pancreatitis in mice, it has been shown that mice with deletions of RIP3 or MLKL are highly resistant to cerulein pancreatitis. ${ }^{32,33}$ These findings provide further verification that acinar cell death in murine cerulein pancreatitis is mediated by necroptosis rather than by apoptosis. Whether a similar situation obtains in other forms of murine experimental pancreatitis or in human pancreatitis remains to be seen. This is not a trivial question since the form of cell death occurring in pancreatitis could determine a unique approach to the treatment of the disease. It should be noted, however, that both forms of cell death are initiated by TNF- $\alpha$, indicating that generation of this cytokine during the inflammation can mediate either apoptosis or necroptosis and in this sense, pancreatitis in different species and in different models may be similar.

Recently it has been shown that necroptosis is also involved in pancreatic oncogenesis in that expression of RIP 1 and RIP 3 is elevated in human pancreatic ductal adenocarcinoma and inhibition/deletion of these components lead to decreased tumor progression. ${ }^{34}$ This may relate to the fact that RIP1/ RIP3-induced necroptosis induces CXCL1 production and SAP130 activation of Mincle, which then leads to the accumulation of myeloid-derived suppressor cells and Mincle-stimulated dendritic cells in the tumor environment that suppress $T$ cells with anti-tumor activity. Whether this complex mechanism influences pancreatitis progression is currently unknown.

\section{INITIATION OF INFLAMMATION IN PANCREATITIS BY STIMULATION OF INNATE IMMUNE RESPONSES BY DAMAGE-ASSOCIATED MOLECULAR PATTERNS (DAMPS) AND NEUTROPHIL-EXTRACELLULAR TRAPS (NETS)}

Given the fact that the uninflamed pancreas is a sterile organ, the initial cellular injury (as discussed above, most likely triggered by pathological trypsinogen activation) occurs in an aseptic environment (Figure 1). Studies of the inflammation nevertheless strongly suggest that it is likely to result from the release of damage-associated molecular patterns (DAMPs) from dying pancreatic acinar cells that then induce inflammation mediated by activation of PRRs (pattern recognition receptors) of the innate immune system. ${ }^{35}$ Various kinds of DAMPs, including high-mobility group box protein 1 (HMGB1), self-DNA, nucleosomes (DNA coiled around histone octamers) and adenosine triphosphate have in fact been identified as pancreatitis-associated "danger signals" (i.e., DAMPs) in human and experimental pancreatitis. ${ }^{36,37}$ Of these, HMGB1 is of particular interest since Yasuda et al., reported markedly elevated levels of this DAMP in both animals and patients with acute pancreatitis ${ }^{38,39}$ and Sawa et al., ${ }^{40}$ reported attenuation of experimental pancreatitis is achieved by administration of neutralizing antibodies to HMGB1. It should be noted, however, that intracellular HMGB1 inhibits inflammatory nucleosome release (see discussion below) and may have an inhibitory effect on pancreatitis as an intracellular protein. ${ }^{37}$

Hoque et al. have elucidated a possible molecular mechanism mediated by DAMPs-PRR interaction in cerulein-induced pancreatitis. These authors showed that during the early phase of cerulein-induced pancreatitis, self-DNA released from the dying acinar cells activates pancreatic macrophage TLR9, an endosome-linked PRR responsive to bacterial CPG DNA and self-DNA. ${ }^{35,41,42}$ In parallel with this event, released adenosine triphosphate induces the activation of $\mathrm{P}_{2} \mathrm{X}_{7}$, a purinergic receptor, which then acts in concert with TLR9 activation to trigger the activation of the NLRP3 inflammasome and the conversion of the pro-IL-1 $\beta$ to mature IL- $1 \beta$.

Very recently, a DAMP-PRR interaction in pancreatitis involving the AIM2 (absent in melanoma 2) inflammasome has 


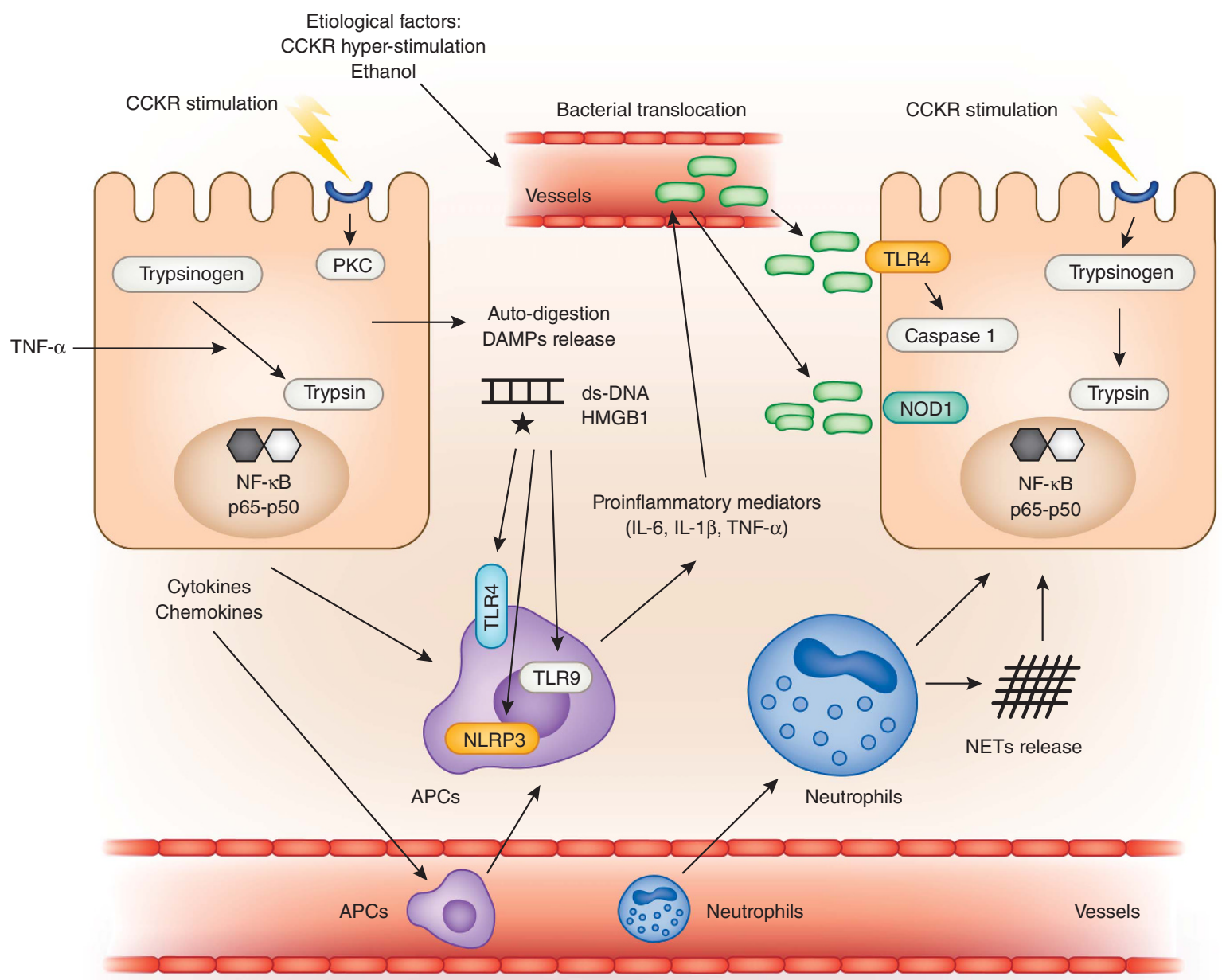

Figure 1 Initiating events in the induction of pancreatitis: acinar cell injury and release of DAMPs. This figure depicts the initiating events that result in pancreatitis. A variety of factors, most notably CCKR hyper-stimulation caused in one experimental model by cerulein and perhaps in humans by excessive ethanol ingestion leads via PKC signaling to dysregulation of intracellular acinar proteases and the generation of trypsin from trypsinogen. This causes acinar cell injury and release of DAMPs such as host dsDNA and HMGB1 that then induce APCs in the vicinity of the acinar cells via various TLRs and NLRP3 to produce a mixture of pro-inflammatory cytokines. An important consequence of such release is the induction of changes in intestinal permeability and the translocation of gut commensal microflora into the circulation, where the latter stimulates acinar cells via TLR4 and NOD1. TLR4 signaling leads to the activation of the NLRP3 inflammasome and the production of caspase 1 and IL-1 $\beta$. NOD1 signaling in cooperation with CCKR signaling results in the activation of NF- $\kappa \mathrm{B}, \mathrm{TNF}-\alpha$, and type I IFN factors that sustain the inflammation as shown in Figure 2 . Another early feature of pancreatitis is the migration of neutrophils into the pancreas, where they enhance trypsinogen activation and cell damage either directly or indirectly via the generation of NETs. APCs, antigen-presenting cells; CCKR, cholecystokinin receptor; DAMPs, damage-associated molecular patterns; dsDNA, double-stranded host DNA; NETs, neutrophil extracellular traps; NF-кB, nuclear factor-kappa B; NLRP3, NOD-like receptor family pyrin domain containing-3; NOD1, nucleotide-binding oligomerization domain 1; PKC, protein kinase C; TLRs, toll-like receptors.

been reported. In this case, the stimulating DAMPs were nucleosomes mentioned above as possible pro-inflammatory DAMPs in pancreatitis. In the relevant studies, Kang et al. ${ }^{43}$ provided evidence that nucleosome stimulated the AIM2 inflammasome (and IL-1 $\beta$ production) but that such stimulation required dsRNA-activated protein kinase phosphorylation mediated by RAGE (receptor for advance glycation end products). In addition, they found that both AIM2- and RAGE-deficient mice exhibit reduced L-arginineinduced pancreatitis as well as reduced IL-1 $\beta$ and HMGB1 expression, the latter an inflammasome product, under these circumstances.
Another factor contributing to the initial inflammation of pancreatitis relates to the observation discussed above that stimulation of the innate immune system by DAMPs results in inflammasome activation and the release of IL- $1 \beta$, the latter a potent neutrophil chemotactic factor via its upregulation of leukocyte adhesion molecules. ${ }^{44}$ This observation introduced the possibility that the early neutrophil infiltration characteristic of pancreatitis is both a result and cause of initial pancreatic inflammation.

Initial evidence for the latter possibility came from studies showing that neutrophil infiltration of the pancreas following pancreatitis induction is an early phenomenon and that such 
infiltration results in trypsinogen activation. ${ }^{45,46}$ Insight into the mechanism of such neutrophil-mediated trypsinogen activation came from subsequent studies showing that taurocholate induction of acute pancreatitis in mice induces formation of extracellular fibrillar and web-like structures, which disappear with treatment with DNase. ${ }^{47}$ Such structures were highly reminiscent of neutrophil-extracellular traps (NETs), web-like structures composed of extracellular DNA, chromatin, molecules derived from granules ${ }^{48,49}$ and it was thus apparent that neutrophil-derived NETs might be causing trypsinogen activation. Evidence for this possibility has come from the observation that inhibition of NETs formation by the administration of DNase protects mice from pancreatitis induced by taurocholate or L-arginine. Moreover, plasma levels of DNA-histone complexes, i.e., NETs elements, are markedly elevated in patients with acute pancreatitis. Finally, Merza et al. have shown that NETs released from neutrophils are potent in vitro activators of trypsinogen in acinar cells. Taken together, these results suggest that initial pancreas injury caused by trypsinogen activation induces the migration of neutrophils into the pancreas and this in turn causes further trypsinogen activation via generation of NETs.

The acinar cell death resulting from the release of DAMPs in the early stages of pancreatitis discussed above is a prelude to the translocation of commensal organisms into the circulation and their subsequent stimulation of innate immune responses in acinar cells that sustain the inflammation, as discussed below. An interesting side-light to this phenomenon is the fact that dendritic cells might serve as a break on this phenomenon via their capacity to clear cellular necrotic debris. This is suggested by the fact that depletion of DCs results in intensification of experimental pancreatitis and decreased acinar cell viability. ${ }^{50}$

\section{INVOLVEMENT OF MICROBE-ASSOCIATED MOLECULAR PATTERNS (MAMPS) AND PATHOGEN RECOGNITION RECEPTORS (PRRS) IN PANCREATITIS}

So far in our discussion of the pathogenesis of pancreatitis we have been concerned mainly with early intra-acinar cell events that are involved in the initiation of inflammation (although these events may be persistent and contribute to ongoing inflammation as well) (Figure 1). However, pancreatitis is unique in that the inflammation leads rapidly to changes in the permeability of the bowel wall that allow translocation of intraluminal bacteria into the circulation and therefore the exposure of acinar cells to pro-inflammatory effects of organisms in the gut microbiome. ${ }^{51}$ As we shall see, this may be the major driving force of pancreatic inflammation after its initiation.

The first hint that this is in fact the case came from clinical studies which showed that severe pancreatitis can lead to local and extra-pancreatic complications such as pancreatic necrosis or systemic inflammatory response syndrome or multiple organ dysfunction syndrome, ${ }^{1,5,51,52}$ which were shown to be manifestations of pancreatitis largely due to invasion of pancreatic tissue by gut organisms and subsequent dissemination of the latter to distant organs and/or endotoxemia. ${ }^{51}$
Studies of the types of organisms involved were sporadic and incomplete until Li et al. ${ }^{53}$ conducted a comprehensive study of bacterial invasion in pancreatitis using 16S rRNA-based technology. They found that polymicrobial bacterial DNA can be detected in the circulation of almost $70 \%$ of patients with pancreatitis and this percentage increased with increased disease severity. Most of the organisms were similar to those found in the gastrointestinal tract and therefore had probably originated in the bowel lumen; in addition, while in most cases these bacteria consisted of commensal organisms, at least in some instances, organisms considered to be pathogens were also found. It is presumably the latter that account for the more severe complications of bacterial invasion in pancreatitis such as pancreatic necrosis. Although these studies highlight the prevalence of bacterial translocation from the bowel during pancreatitis, they provide no insight into the mechanism of the altered bowel permeability allowing such translocation. Indeed, the mechanism involved is poorly understood although it may involve the release of unique pancreatitis-specific cytokines that affect gut permeability that are not generated in other forms of gut inflammation.

The high rate at which bacteria are found in the circulation of patients with pancreatitis suggest that these bacteria could have a role in disease pathogenesis that transcends their capacity to cause local and systemic infections in a minority of patients. This concept is supported by findings in several animal studies. First, enteric bacteria have been detected in the mesenteric lymph nodes and the translocation of fluorescent beads having the size of bacteria into the pancreas via the peritoneum has been seen upon induction of experimental pancreatitis. ${ }^{54-56}$ Second, experimental pancreatitis is in fact accompanied by increased intestinal permeability. ${ }^{57}$ Third and finally, bowel sterilization via antibiotic treatment reduces pancreatic inflammation, infection, and mortality in various experimental pancreatitis models. ${ }^{58-61}$

More definitive support for the role of translocated bowel organisms in the pathogenesis of pancreatitis comes from recent studies showing that such translocation is necessary for the development of cerulein-induced pancreatitis. Thus, as shown by Tsuji et al., ${ }^{58}$ antibiotic-induced bowel sterilization rendered mice virtually completely resistant to ceruleininduced pancreatitis but such resistance was negated when the bowel sterilization was accompanied by oral administration of a commensal Escherichia coli expressing LacZ (ECLACZ) that was resistant to the antibiotics in the bowel sterilization regimen. Thus, colonizaton of the gut with a commensal organism was both necessary and sufficient for the induction of cerulein pancreatitis.

These studies of the role of the gut microflora in pancreatitis intermesh with studies examining the role of bacteriaassociated MAMPs and their capacity to stimulate PRRs such as TLRs and nucleotide-binding domain and leucine-rich repeat-containing receptors (NLRs) in the induction of experimental pancreatic inflammation. ${ }^{42,62,63}$ Studies of the role of TLR4, the prototypical PRR that responds to LPS derived from Gram-negative bacteria, was the first PRR to be studied. ${ }^{42}$ 
It was found that mice deficient in TLR4 are partially protected from acute cerulein-induced pancreatitis ${ }^{64}$ and severe lung injury associated with pancreatitis is induced by combined treatment of cerulein and LPS as compared with cerulein alone. ${ }^{65}$ As alluded to above, the possible role of TLR4 in causation of pancreatitis has been highlighted in studies of ethanol-induced pancreas injury. ${ }^{16}$ The theory is that an ethanol-induced leaky gut syndrome leads to bacterial translocation into the circulation, followed by sensing of LPS by TLR4 expressed in pancreatic acinar cells and activation of the NLRP3 inflammasome. The resulting production of mature IL- $1 \beta$ then aggravates gut permeability changes and contributes to other aspects of pancreatitic inflammation. Interestingly, a polymorphism in the TLR4 gene has been identified as a susceptible factor for acute pancreatitis. ${ }^{66}$ This raises the question of whether abnormalities of TLR4 structure or level of expression can enhance TLR4-mediated proinflammatory responses leading to pancreatitis in certain individuals.

More impressive studies of the role of a PRR in pancreatitis are those evaluating the role of NOD1 in pancreatitis pathogenesis. ${ }^{58,67}$ NOD1 was originally characterized as a cytosolic PRR that detects small peptide components derived from bacterial peptidoglycan associated with gut pathogens. ${ }^{63}$ As such, NOD1 was shown to have a protective role in mucosal host defense against various kinds of pathogenic organisms such as Shigella flexneri, pathogenic E. coli, and Helicobacter pylori. ${ }^{68-71}$ In later studies, however, it was established that NOD1 is also capable of reacting to peptidoglycan components derived from commensal organisms. This was shown first by the fact that sensing of commensal organisms by NOD1 is required for lymphoid tissue organogenesis. ${ }^{72}$ More importantly to the present discussion, it was more recently shown that NOD1 expression on acinar cells and subsequent interactions between NOD1 at this site with commensal organisms is essential to the development of cerulein-induced pancreatitis in that NOD1-deficient mice are almost completely resistant to the induction of this pancreatitis. ${ }^{58}$

The role of bacterial translocation in experimental pancreatitis was further explored in a model of cerulein-induced pancreatitis in which pancreatitis was induced by administration of a low dose of cerulein (that does not itself induce pancreatitis yet is still capable of acinar cell signaling) and FK156, a ligand and activator of NOD1 that also does not itself induce pancreatitis. ${ }^{58,67}$ This form of cerulein-induced pancreatitis, termed low-dose cerulein pancreatitis was developed to clearly differentiate the respective roles of NOD1 signaling and CCKR signaling in the pathogenesis of the pancreatitis, as will be discussed in more detail below. Using this model, it could be shown that although IP injection of ECLACZ or lowdose cerulein alone (to mice sterilized by antibiotic treatment) does not cause pancreatitis, administration of both of these factors does cause pancreatitis and that the latter is at least partially dependent on the presence of NOD1. Thus, in this low-dose cerulein model, the NOD1 activation by FK156 is taking the place of translocated bacteria expressing a NOD1 ligand. Finally, in studies addressing bacterial translocation in low-dose cerulein pancreatitis, it could be shown that the level of bacteremia was relatively low in mice treated with low-dose cerulein alone and relatively high in mice treated with both lowdose cerulein and FK156; thus a high level of bacteremia (due to bacterial translocation) requires the initiation of pancreatitis. In further studies addressing the same question, mice with bowel sterilization administered increasing oral doses of ECLACZ and then subjected to low-dose cerulein and FK156 administration exhibited a level of pancreatitis proportional to the ECLACZ dose. This suggested that whereas initiation of lowdose cerulein pancreatitis requires both low-dose cerulein and NOD1 ligand, the pancreatitis is ultimately sustained by the bacterial translocation and de novo NOD1 activation caused by the initiated pancreatitis.

Taken together, these results definitively establish that while pancreatic inflammation may be initially triggered by intraacinar events such as trypsinogen activation, it ultimately depends on subsequent immune responses induced by the activation of components of the innate immune system, notably NOD1.

\section{ACTIVATION OF NF- $\chi B$ IN PANCREATITIS}

In the knowledge that innate immune responses at least in part involving NOD1 activation are necessary for induction of cerulein-induced pancreatitis as discussed above and that such activation may lead to NF- $\kappa \mathrm{B}$ activation, one might assume that NF- $\kappa \mathrm{B}$ activation is a central feature of pancreatic inflammation as it is in the case of other major inflammatory diseases. A host of evidence fully supports this conjecture (Figure 2). First, it has been shown that administration of cerulein at doses causing pancreatitis leads to activation of pancreatic NF- $\kappa B$ and that such activation is accompanied by nuclear translocation of p65, p50, and p52 in both gel-shift assays and in immunoblotting studies. ${ }^{73-76}$ Second, this activation occurs pari passu with the transcription and release of NF- $\mathrm{KB}$ pro-inflammatory target genes such as TNF- $\alpha$ and monocyte chemotactic protein1 (MCP-1), indicating that NF- $\mathrm{KB}$ activation is leading to the synthesis of a host of pro-inflammatory factors that have the potential to drive various aspects of the pancreatic inflammation. ${ }^{58,77,78}$ Third, the latter possibility has in fact been established by studies showing that mice deficient in $\mathrm{NF}-\mathrm{\kappa B} / \mathrm{p} 105$, a precursor of $\mathrm{p} 50$, are resistant to ceruleininduced pancreatitis as are mice administered low molecular weight-specific inhibitors of NF- $\kappa$ B activation. ${ }^{79-81}$ Finally, the development of pancreatitis can be induced in animals treated with intra-pancreatic ductal retrograde infusion of adenovirus-mediated gene delivery of the NF- $\kappa \mathrm{B}$ subunit $\mathrm{p} 65 .^{82}$

The relation of NF- $\kappa \mathrm{B}$ activation in pancreatitis to aberrant trypsinogen activation has come from studies of an elegant murine model of pancreatitis established by Baumann et al. ${ }^{83}$ These authors created transgenic mice that regulate the activation of NF- $\mathrm{\kappa B}$ by expression of either a dominant negative (DN) or a constitutive active (CA) form of IKK $\beta$ under the control of a tetracycline-responsive elastase promoter. Thus, in this model one of these forms of IKK $\beta$ is overexpressed 
a

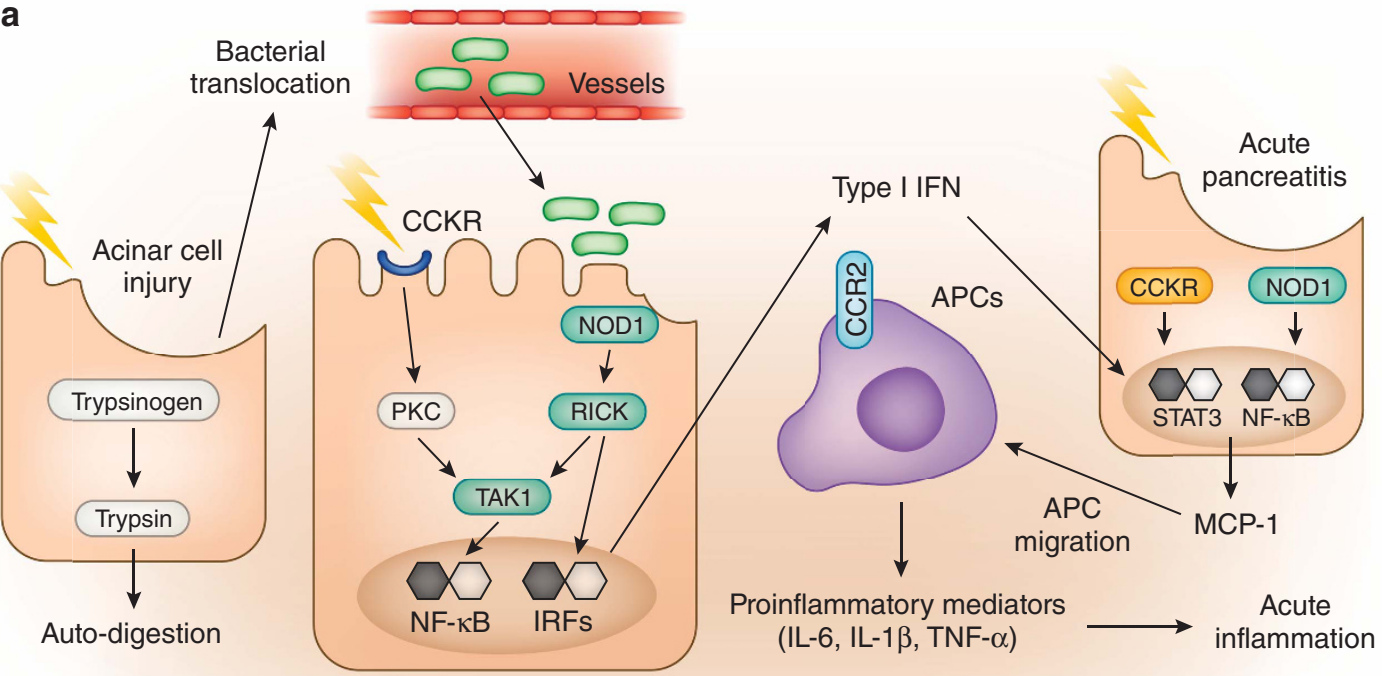

b

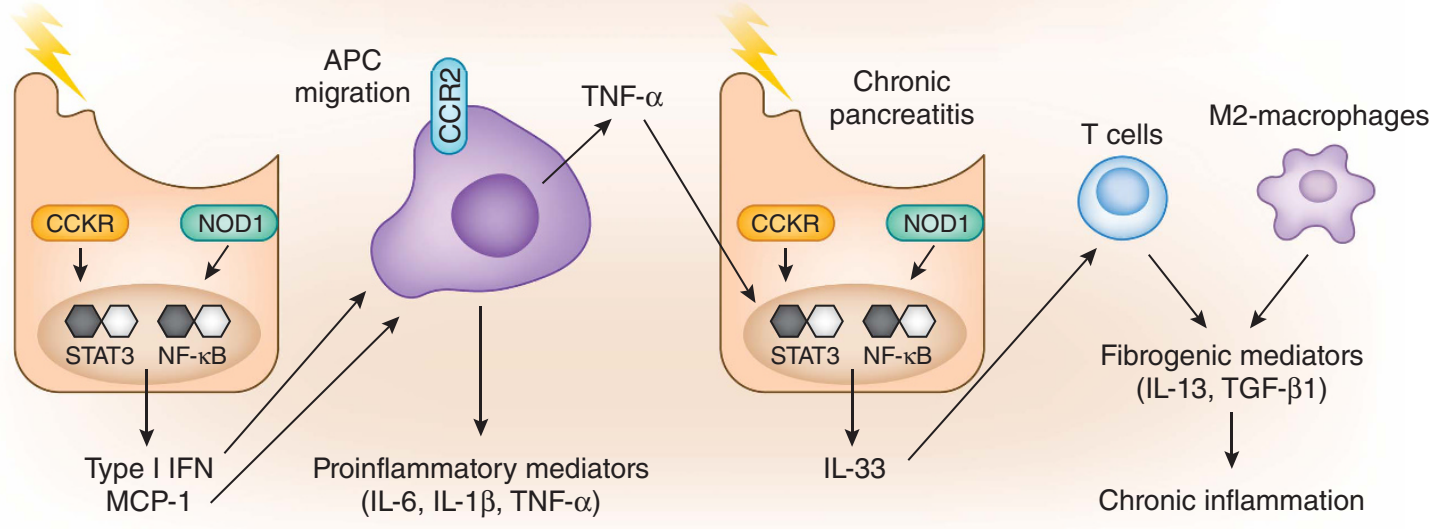

Figure 2 Innate immunological response that sustain pancreatitis and underlie the fibrosis of chronic pancreatitis. This figure depicts the complex series of immunological events that sustain pancreatitis after initial acinar damage and translocation of commensal organisms from the gut into the circulation has occurred, as shown in Figure 1. In this figure, five diagrammatic acinar cells are shown, each roughly representing different stages of pancreatitis pathogenesis. (a) The first acinar cell (top, on the left) represents the first stage that has been shown in greater detail in Figure 1. The top middle and right acinar cells illustrates the fact that signaling via CCKR and NOD1 act in concert to induce activation of NF-KB and IRF and type I IFN expression; these factors then act downstream to induce expression of MCP-1, a chemotactic ligand that draws pro-inflammatory CCR2-bearing macrophages into the pancreas. These macrophages produce a host of pro-inflammatory mediators of the full-blown inflammation; among these are cytokines that support T-cell differentiation into Th1 or Th17 cells. (b) The bottom acinar cells represent cells that occur in the pancreas during chronic pancreatitis that have subjected to apoptotic or necrotic cell injury due to secretion of TNF- $\alpha$ by macrophages. This cell injury leads to the generation of IL-33, an alarmin cytokine that acts via the ST2 receptor on T cells to induce fibrogenic mediators, particularly IL-13. The latter may then act on M2-macrophages to induce production of TGF- $\beta 1$ and other downstream fibrogenic factors. This pro-fibrotic program underlies the development of fibrosis that is characteristic of chronic pancreatitis. CCKR, cholecystokinin receptor; CCR2, C-C chemokine receptor type 2; IFN, interferon; IRF, interferon regulatory factor; MCP-1, monocyte chemotactic protein-1; NOD1, nucleotide-binding oligomerization domain 1; NF-кB, nuclear factor-kappa B; TNF, tumor necrosis factor.

exclusively in acinar cells when the mouse is administered tetracycline and this, in turn, leads to either suppressed or activated NF- $\kappa B$ signaling depending on whether DN-IKK $\beta$ and CA-IKK $\beta$ is being overexpressed, respectively. ${ }^{83}$ Using these transgenic mice, Baumann et al. showed first that expression of CA-IKK $\beta$ in acinar cells leads to spontaneous development of severe pancreatitis, as was the case in the aforementioned studies of intra-ductal adenovirus administration of $\mathrm{p} 65$, whereas in contrast, the expression of DN-IKK $\beta$ in acinar cells suppresses cerulein-induced pancreatitis. Furthermore, they showed that overexpression of either
DN-IKK $\beta$ or CA-IKK $\beta$ had no significant effect on cerulein-induced acinar cell trypsinogen activation. Taken together, these studies indicate that NF- $\mathrm{\kappa B}$ activation in acinar cells is indispensable for the development of pancreatitis and that CCKR-mediated trypsinogen activation cannot induce pancreatitis in the absence of such activation.

The above studies showing the importance of NF- $\kappa B$ activation in the pathogenesis of pancreatitis do not address the question of whether trypsinogen activation and NF- $\kappa \mathrm{B}$ activation are interrelated or independent events. Several reports show that one of the signaling outcomes of CCKR over- 
stimulation is the activation of NF- $\mathrm{KB}$ via a PKC pathway ${ }^{84}$ or a phosphatidylinositol 3-kinase (PI3K) pathway ${ }^{85}$ and that pharmacological inhibitors of PKC or PI3K block CCKR induction of NF- $\mathrm{KB}$ in pancreatic acinar cells. In addition, blockade of intracellular $\mathrm{Ca}^{++}$influx by a chelator also inhibits NF- $\kappa B$ induction owing to the fact that PKC-mediated NF- $\kappa B$ activation in this model requires such influx. ${ }^{18,73,74,84,86}$ These findings, however, do not necessarily imply that the other major outcome of CCKR stimulation, trypsinogen activation and trypsin expression, results in NF- $\kappa \mathrm{B}$ activation. That it does not was nicely shown by the fact that acinar cell expression of an intracellular mutant trypsinogen subject to conversion to intracellular trypsin by an endogenous protease did not induce NF- $\kappa$ B activation. ${ }^{87,88}$ Thus, the conclusion that can be drawn from these various studies is that whereas CCKR overstimulation does indeed induce both NF- $\kappa \mathrm{B}$ activation and trypsinogen activation, these two events are independent. ${ }^{73} \mathrm{An}$ additional aspect relating to the outcome of CCKR signaling is that the induction of NF- $\kappa B$ activation is accompanied by the induction of TNF- $\alpha$ production. As discussed below, this important attribute of CCKR signaling plays into the mechanism of acinar cell damage mediated by infiltrating macrophages.

If indeed CCKR over-stimulation leads to NF- $\kappa \mathrm{B}$ activation and the latter is sufficient for the development of pancreatitis as implied by the Bauman et al., studies discussed above, why is it that high-dose administration of cerulein is not in itself capable of inducing acute pancreatitis in mice subjected to bowel sterilization and are not able to develop either acute or chronic pancreatitis in the absence of NOD1? A possible answer to this important question came from signaling studies obtained from mice subjected to the low-dose cerulein-induced pancreatitis developed by Tsuji et al. alluded to above in which the pancreatitis had been induced by administration of a low dose of cerulein together with a NOD1 ligand, neither of which is able to induce pancreatitis when administered alone. ${ }^{58,67}$ The acinar cells from the pancreatitis in these mice were attractive targets of signaling studies because they suggested that whereas neither low-dose cerulein nor NOD1 ligand can induce a sufficient amount of NF- $\kappa \mathrm{B}$ to support pancreatitis when administered alone these stimuli can do so when administered together.

In the relevant studies, it was found that administration of a low dose of cerulein alone resulted in acinar cell PKC activation followed by the interaction between PKC and TGF- $\beta$-activated kinase (TAK1). ${ }^{58}$ However, such TAK1 activation was not sufficient for induction of NF- $\kappa \mathrm{B}$. On a separate track, administration of NOD1 ligand alone activated acinar cell receptor-interacting serine-threonine kinase (RICK), its immediate downstream signaling partner, but such RICK activation was again not itself sufficient for the activation of NF$\kappa B$. In contrast, simultaneous activation of CCKR and NOD1 by a low dose of cerulein and NOD1 ligand led to interaction between RICK and TAK1 as well as substantial activation of NF- $\kappa B .^{58}$ These findings thus implied that cerulein signaling via CCKR and NOD1 activation acts in a cooperative fashion to induce NF- $\mathrm{KB}$ during the induction of pancreatitis. With respect to the fact that high-dose cerulein administration cannot induce pancreatitis in the absence of NOD1 signaling, the above results are most consistent with the view that despite in vitro studies showing that CCKR over-stimulation can give rise of NF- $\mathrm{KB}$ activation, such stimulation is, in reality, an inadequate inducer of pancreatitis in the absence of NOD1 stimulation. On the other hand, it can give rise to a sufficient initial acinar cell DAMPs response and induction of cytokines that cause changes in bowel permeability and entry of NOD1stimulating microflora. Finally, it is important to point out that, as discussed below, NOD1 signaling is essential to the development of pancreatitis by mechanisms that involve $\mathrm{NF}-\mathrm{\kappa B}$ and signal tranducer and activator of transcription 3 (STAT3) signaling; it is therefore reasonable to postulate that even if CCKR over-stimulation is sufficient to cause NF- $\kappa B$ activation by itself, such activation alone is not sufficient for the development of pancreatitis.

\section{NF- $\chi$ B/STAT3-MEDIATED MCP-1 INDUCTION AND MACROPHAGE INVASION IN PANCREATITIS}

Activation of NF- $\kappa B$ in cerulein-induced pancreatitis by the mechanisms discussed above is an essential component of the mechanisms of inflammation driving this disease (Figure 2). One of the most important of these mechanisms (to which we will now turn our attention) is that underlying the induction of CCL2 (MCP-1), a chemokine necessary for the influx of inflammatory macrophages into the pancreas.

In studies of low-dose cerulein pancreatitis addressing this issue, it was found that NOD1 stimulation by its ligand FK156, in combination with low-dose cerulein administration induces MCP-1 as well as IL-6 production and, as before, such NOD1 stimulation mimics the effect of IP administration of commensal bacteria (e.g., ECLACZ) ${ }^{58}$ In addition, NOD1/ low-dose cerulein administration causes the pancreatic infiltration of large numbers of $\mathrm{CD}_{11 \mathrm{~b}^{+}}$myeloid cells bearing $\mathrm{C}-\mathrm{C}$ chemokine receptor type 2 (CCR2). This was in accord with previous studies that have shown that MCP-1 has a role in the macrophage-dependent inflammatory response characterizing pancreatitis. ${ }^{89}$ In further studies of the low-dose cerulein pancreatitis model it was shown that CCR2-deficient mice exhibit decreased evidence of pancreatitis along with decreased infiltration of $\mathrm{CD} 11 \mathrm{~b}^{+}$macrophages. ${ }^{58}$ In addition, studies of bone marrow chimeras allowed one to establish that NOD1bearing non-hematopoietic cells (i.e., acinar cells) were necessary for the development of pancreatitis as well as the induction of MCP-1 in this model. Finally, studies of isolated pancreatic acinar cells from untreated mice showed that NOD1 stimulation was sufficient to induce substantial amounts of MCP-1 secretion but that the latter was augmented by low-dose cerulein stimulation. Together, these studies established that MCP-1 production is an essential initial step in the induction of cerulein-induced pancreatitis and that such production is dependent on NOD1/cerulein stimulation of acinar cells.

The above findings set the stage for studies defining the signaling pathways underlying MCP-1 induction. In initial 
studies along these lines the findings of Deshmane et al..$^{90}$ showing that STAT3 and NF- $\kappa B$ activation underlies induction of MCP-1 were verified with studies showing that expression of phospho-STAT3 (pSTAT3) and phospho-IкB $\alpha(\mathrm{pI \kappa B} \alpha)$ was present in the pancreas of NOD1-intact mice treated with NOD1 ligand/low-dose cerulein but was not seen in the pancreas of NOD1-deficient mice. In addition, these findings were confirmed by studies of bone marrow chimeras either expressing NOD1 or not expressing NOD1 in acinar cells. ${ }^{58}$ Finally, the role of STAT3 and NF- $\mathrm{KB}$ in MCP-1 induction was explored with specific inhibitors of these factors. It was found that administration of JSI-124, a specific inhibitor of STAT3 in combination with IMD-0354, a NF- $\mathrm{BB}$ inhibitor led to complete prevention of low-dose cerulein pancreatitis and MCP-1 production. It was thus evident that MCP-1 production was dependent on signaling mediated by both STAT3 and NF- $\kappa \mathrm{B}$.

In further exploration of MCP-1 induction in low-dose cerulein-induced pancreatitis the mechanism of STAT3 activation in this model was examined. As mentioned above, one outcome of NOD1/low-dose cerulein administration to mice was pancreatic production of IL-6, a cytokine known to induce STAT3 activation and, therefore, potentially involved in MCP-1 production. That this was in fact the case was established with studies showing that MCP-1 production by NOD1/low-dose cerulein stimulated acinar cells was partially blocked by the neutralization of the IL- 6 signaling pathway. ${ }^{58}$

A second potential mechanism of STAT3 activation in this model was suggested by the fact that NOD1 was known to have the unique ability to induce type I IFN, a factor that causes STAT3 (as well as STAT1) activation. ${ }^{58}$ Such induction of type I IFN by NOD1 had been shown to be due to its ability to activate a multistep signaling pathway involving first the activation of interferon regulatory factor 7 (IRF7) and second the priming of the IFN-stimulated gene factor 3 pathway (ISGF3) by IRF7induced type I IFN via the IFN $\alpha \beta R$ (IFNAR) ${ }^{71}$ Indeed, it could be shown that pancreatitis in the NOD1/low-dose cerulein model was attenuated in mice lacking IFNAR and this was associated with both reduced STAT3 and MCP-1 expression. It should be noted that low-dose cerulein also induces STAT3 via CCKR-activation of janus activating kinase $2,{ }^{91}$ so that both NOD1 and cerulein signaling again act cooperatively to induce STAT3 activation, albeit by a different mechanism than that governing NF- $\mathrm{KB}$ induction.

Taken together, these various studies established that STAT3 activation in low-dose cerulein pancreatitis (and by extension in pancreatitis in general) is a complex process involving both IL-6 induction mainly due to NF- $\kappa \mathrm{B}$ activation and type I IFN production mainly due to NOD1 activation. Although the essential outline of the signaling pathways have been defined in the acute model of low-dose cerulein pancreatitis, similar pathways probably apply to the chronic model of cerulein pancreatitis wherein low-dose cerulein and NOD1 ligand is administered for a more prolonged period. ${ }^{67}$ This is evident from the fact that mice deficient in IFNAR are resistant to the development of chronic pancreatitis. Finally, it is important to point out that whereas STAT3 activation has a central role in pancreatitis development in relation to induction of MCP-1 and inflammatory macrophage infiltration it also has a role in the inflammation via its involvement of cytokines that contribute to the inflammation such as type I IFN and IL- $6 .{ }^{67}$

As discussed above, there is compelling evidence that activation of NF- $\mathrm{KB}$ and STAT3 in acinar cells is a central event for the development of experimental (murine) pancreatitis. However, our knowledge regarding the activation status of these transcription factors in human pancreatitis is very limited and somewhat paradoxical. Analysis of the status of NF- $\kappa B$ and STAT3 in the peripheral blood cells but not the pancreatic tissue of pancreatitis patients, provided unexpected evidence that activation of NF- $\mathrm{\kappa B}$, STAT1, and STAT3 is reduced in acute pancreatitis patients as compared with those from healthy controls. ${ }^{92-94}$ The molecular mechanisms accounting for such reduced activation are not fully understood, but could involve tolerogenic responses to MAMPs expressed by translocated intestinal organisms. Thus, tolerance to MAMPs may be one possible mechanism accounting for reduced innate immune responses to the same or other MAMPs in pancreatitis. ${ }^{95}$

\section{THE ROLE OF TYPE I IFN IN PANCREATITIS}

Mice deficient in IFNAR and thus unable to signal via type I IFN are resistant to both the development of acute low-dose cerulein-induced pancreatitis (as already mentioned) as well as chronic low-dose cerulein-induced pancreatitis ${ }^{58,67}$ (Figure 2). Although this is partly explained by its role in the induction of STAT3 and MCP-1 in acinar cells, it also is due to various effects of this cytokine on macrophage effector function. The role of type I IFN in macrophage effector function is immediately evident from studies showing recruitment and maturation of the Ly $6^{\text {hi }}$ monocytes is uniquely dependent on type I IFN induction of CCR2 expression, which, as noted above, is the receptor for MCP-1. ${ }^{96}$ Thus, type I IFN is responsible for both the receptor and ligand aspects of the chemotaxis mediating macrophage infiltration of the pancreas.

Yet another role of type I IFN in relation to macrophage effector function in pancreatitis relates to evidence that it induces macrophage TNF- $\alpha$ production. As noted in the discussion above, myeloid cells in cerulein-induced pancreatitis cause necroptotic acinar cell death via production of TNF- $\alpha$. Evidence that type I IFN is involved in such production came from studies of pancreatic cells extracted from mice with chronic cerulein-induced pancreatitis wherein it was observed that pancreatic acinar cells co-cultured with pancreatic $\mathrm{CD} 11 \mathrm{~b}^{+}$macrophages release greatly increased amounts of IL-33, a cytokine associated with acinar cell death (see discussion below) and that such IL-33 release is inhibited by anti-IFNAR Ab (presumably blocking type I IFN stimulation of the macrophages) or anti-TNF- $\alpha$ Ab (presumably blocking TNF- $\alpha$ stimulation of acinar cells). ${ }^{67}$ The capacity of type I IFN to induce macrophage TNF- $\alpha$ production is supported by a prior study by Mancuso et al. ${ }^{97}$ showing that such production induced by live bacteria is mediated by type I IFN signaling. 


\section{PRO-INFLAMMATORY CYTOKINE ACTIVITY IN PANCREATITIS ARISING FROM INNATE IMMUNE RESPONSES}

The basic picture of pancreatitis pathogenesis suggested above is that acinar cell damage initiated by various causes of dysregulated trypsinogen activation leads to the release of DAMPs that then react with PRRs on antigen-presenting cells (APCs) to cause the generation of cytokines that induce a change in bowel wall permeability (Figure 2). This, in turn, leads to the entry of commensal organism into the circulation and the activation of NOD1 (and possibly TLR4 as well) that, in concert with CCKR signaling causes activation of $N F-\kappa B$, a type I IFN response and ongoing pro-inflammatory cytokine elaboration by PRR-expressing (but as yet poorly defined) APCs responsive to DAMPs and MAMPs. ${ }^{35,42,63}$ Consistent with this scenario, patients with acute pancreatitis exhibit elevated serum levels of various pro-inflammatory cytokines such as IL-6, IL-1 $\beta$, and TNF- $\alpha,{ }^{98-100}$ and elevated serum levels of various chemokines such as IL-8, MCP-1, and macrophage migration inhibitory factor, which presumably arise from activated cells in the pancreas. ${ }^{100-102}$

These "generic" pro-inflammatory factors drive (or aggravate) the pancreatic inflammation in various specific and/or nonspecific ways. For instance, IL-6, one of the prototypical inflammatory cytokines, may enhance inflammation by its role in the generation of pathological T helper type 17 (Th17) cells or, as discussed above, its contribution to the induction of MCP-1 and the entry of inflammatory macrophages into the pancreas. In any case, it has been shown that administration of anti-IL-6 Ab to mice with pancreatitis induced by cerulein and LPS leads to marked attenuation of the pancreatitis as well as accompanying pulmonary inflammation. ${ }^{103}$ It should be noted, however, that IL-6 can have anti-inflammatory effects as well. This is shown by the fact that mice genetically deficient in IL-6 exhibit more severe cerulein-induced pancreatitis as compared with IL-6-intact mice. ${ }^{104}$ This contrary finding may be due to the fact that a genetic deletion of IL- 6 leads to profound disruption of pro-inflammatory machinery necessary for pancreas homeostasis, than does-acute administration of anti-IL-6 Ab.

IL-1 $\beta$ is a second prototypic pro-inflammatory cytokine associated with the pathogenesis of human acute pancreatitis. ${ }^{98}$ This cytokine could be mediating pancreatic inflammation in multiple ways including its ability to induce neutrophil infiltration into sites of inflammation and its more general effect on the induction of other pro-inflammatory cytokines and chemokines. ${ }^{36}$ Interestingly, there is evidence that a precursor form of this cytokine is induced by DAMPs acting via TLR9, suggesting that IL- $1 \beta$ may have a role in the induction of enhanced gut permeability to commensal organisms. ${ }^{41}$ The precursor form of IL- $1 \beta$ is converted into an active form via the NLRP3 inflammasome and thus mice deficient in NLRP3 inflammasome component are resistant to the induction of cerulein-induced pancreatitis. ${ }^{41,62}$ Moreover, attenuation of experimental pancreatitis in the absence of TLR9 is accompanied by the presence of pancreatic APCs with impaired production of IL- $1 \beta$ as well as pro-IL- $1 \beta$, suggesting that IL-1 $\beta$ has an autocrine effect on its own synthesis. ${ }^{41}$

TNF- $\alpha$ is a third prototypic pro-inflammatory cytokine involved in pancreatitis pathogenesis and one that appears to have a central role in experimental pancreatitis. As discussed above, this cytokine is induced in pancreatic APCs and is therefore a prime suspect for causing the initial injury to acinar cells occurring in response to excessive CCKR stimulation. This possibility has recently been supported by Sendler et al. ${ }^{105}$ who showed that stimulation of pancreatic acinar cells by TNF- $\alpha$ causes direct activation of pancreatic enzymes and thus leads to premature protease activation and cell necrosis. Type I IFN may also have an important pathological role by its ability to induce CCR2, the chemokine receptor attracting pro-inflammatory macrophages expressing TNF- $\alpha$ into the pancreas, as already discussed above. These macrophages are cellular source of TNF- $\alpha$ in the inflamed pancreas of cerulein-induced pancreatitis ${ }^{106,107}$ and, as also discussed above, may be the important driver of sustained acinar cell necroptosis. These various ways in which TNF- $\alpha$ acts to facilitate pancreatic inflammation provide ample explanation for the fact that the administration of infliximab (monoclonal TNF- $\alpha$ antibody) ameliorates cerulein-induced pancreatic inflammation. ${ }^{106}$ Thus, it is clear that TNF- $\alpha$ has a critical role in the development of experimental pancreatitis. However, confirmation that this cytokine has an equally important role in human pancreatitis awaits the studies of human cells obtained from the inflamed pancreas as well as studies addressing the therapeutic effect of infliximab (anti-TNF- $\alpha$ Ab) administration to patients.

Recently it has become evident that yet another proinflammatory cytokine, IL-33, is an essential element in the pathogenesis of pancreatitis. IL-33 is an "alarmin" cytokine released from dying cells. ${ }^{108}$ Therefore, it is not surprising that serum levels of IL-33 are elevated in patients with severe acute pancreatitis, a condition associated with massive pancreatic acinar cell death. ${ }^{109}$ The role of IL-33 in pancreatitis was extensively analyzed in studies of the chronic model of pancreatitis described by Watanabe et al., ${ }^{67}$ in which, as discussed above, chronic pancreatitis is induced by repeated administration of NOD1 ligand and a low dose of cerulein. This model was of particular relevance for the study of IL-33 because it is marked by the development of fibrosis, a feature of chronic pancreatitis that is driven by IL-33.

The important finding that emerged from these studies was that blocking of IL-33 activity by administration of antibody neutralizing the IL-33 receptor (ST2) to mice subjected to the chronic pancreatitis regimen led to reduction in pancreatic infiltration of immune cells and fibrosis accompanied by decreased pancreatic expression of both pro-inflammatory mediators such as IL-6, TNF- $\alpha$, and MCP- 1 and pro-fibrogenic mediators such as IL-13 and TGF- $\beta 1 .{ }^{67}$ It was thus apparent that IL-33 production has a major role in the pathogenesis of chronic pancreatitis.

In further studies of IL-33 using this model, the source of the IL-33 was probed with bone marrow transplantation studies, 
wherein it was found that induction of chronic pancreatitis was accompanied by IL-33 expression in irradiated NOD1-intact mice transplanted with NOD1-deficient BM cells, but not in irradiated NOD1-deficient mice transplanted with NOD1intact BM cells; thus, these studies suggested that NOD1 stimulation of non-hematopoietic cells, most likely pancreatic acinar cells were the probable source of the IL-33. ${ }^{67}$ In related studies it was found that such acinar cell IL-33 production required the presence of intact type I IFN signaling since mice deficient in IFNAR exhibited a marked decrease in such production as well as resistance to the development of chronic pancreatitis. ${ }^{67}$ This correlates with the fact that, as described above, type I IFN induces TNF- $\alpha$ production by infiltrating macrophages and, in addition co-culture of acinar cells with TNF- $\alpha$-producing $\mathrm{CD}_{11 \mathrm{~b}}{ }^{+}$APCs obtained from inflamed pancreatic tissue is associated with enhanced IL-33 production. Overall, these findings are compatible with the view that pathological macrophages producing TNF- $\alpha$ under the influence of type I IFN induce acinar cell death and release of IL-33. Then, as suggested by studies conducted by Kempuraj et al., ${ }^{110}$ such IL-33 release induces production of pro-inflammatory cytokines by intact acinar cells.

Also relevant to the role of IL-33 in pancreatitis is the recent finding by Xue et al. ${ }^{111}$ that alternatively activated M2 macrophages are numerous in chronic pancreatitis in humans and mice with chronic cerulein-induced pancreatitis. Such M2macrophages bear surface IL- $4 \mathrm{R} \alpha$ and are induced by IL- 4 and IL-13; they are thus likely to be regulated by IL-33 since the latter induces T cells producing IL-13 via the ST2 receptor (see discussion below). These M2-macrophages also produce IL-10 and TGF- $\beta$, the latter a cytokine that may act (in concert with other cytokines) on pancreatic stellate cells that contribute to pancreatic fibrosis via production of extracellular matrix proteins. ${ }^{11,112}$ The importance of these cells in chronic pancreatitis was shown by the fact that pharmacological inhibition of IL- 4 and IL-13 by a blocking peptide led to reduced fibrosis in the cerulein-induced chronic pancreatitis model.

Finally, it should be noted that the above rather definitive studies showing that IL-33 is a major mediator of pancreatitis are opposed by data from several studies showing that under some condition the IL-33-ST2 signaling pathway has a protective role in that mice lacking expression of the ST2 receptor develop enhance pancreatitis. ${ }^{113,114}$ Thus, additional study of IL-33 effects in pancreatitis is warranted.

\section{PRO-INFLAMMATORY CYTOKINE ACTIVITY IN PANCREATITIS ARISING FROM ADAPTIVE IMMUNE RESPONSES}

Adaptive immune responses comprised mainly of T-cell responses to specific antigens also contribute to the pathogenesis of pancreatitis (Figure 2). As in the case of human colitis or murine experimental colitis models these can consist of either excessive effector Th cell function or deficient regulatory $\mathrm{T}$-cell (Treg) function. ${ }^{115,116}$
Several studies of pancreatitis in humans suggest the involvement of Th1 responses in that peripheral blood cells obtained from patients with acute and chronic pancreatitis produce large amounts of prototypical Th1 cytokines, IFN- $\gamma$ and IL-12, and serum levels of IFN- $\gamma$ in such patients are significantly higher than those in healthy controls. ${ }^{117-119}$ This evidence that pancreatitis is associated with a Th1 response is verified by the presence of T cells producing IFN- $\gamma$ in necrotic pancreatic tissue from patients with acute pancreatitis. In contrast to the above human studies, IFN- $\gamma$ has a protective rather than a pathogenic role in cerulein-induced acute pancreatitis since in this case IFN- $\gamma$-deficient mice exhibit increased cerulein-induced pancreatic injury and $\mathrm{NF}-\kappa \mathrm{B}$ activation is suppressed by IFN- $\gamma \cdot{ }^{120}$ Given the fact that a pathological Th17 response is suppressed by IFN- $\gamma$ through downregulation of IL-23 receptor, ${ }^{121,122}$ this protective role of the Th1 response in experimental pancreatitis might be due to the suppression of a pathogenic Th17 response. Compatible to this idea, recent reports support the possible involvement of IL-17 in human acute pancreatitis. ${ }^{123,124}$ In any case, the reason that human and mouse studies regarding Th1 responses are discrepant is unknown.

Studies of experimental models of chronic pancreatitis suggest that Th2 responses also contribute to the pathogenesis of pancreatitis. The most compelling data in this regard comes from the aforementioned study of chronic pancreatitis induced by repeated administration of NOD1 ligand and low-dose cerulein conducted by Watanabe et al. ${ }^{67}$ These authors provided evidence that IL-33 produced by acinar cells acting via the ST2 receptor induces the production of IL-13 and the latter then induces fibrosis via induction of fibrogenic factors such as TGF- $\beta 1$. This is supported by the fact that neutralization of IL-13 by the administration of anti-IL-13 Ab prevented the development of fibrosis but not inflammation in the chronic pancreatitis model, whereas inhibition of IL-33 by administration of anti-ST2 Ab (as noted above) prevented both fibrosis and inflammation. The source of the IL-13 was mainly Th2 cells since pancreatic $\mathrm{CD} 4{ }^{+} \mathrm{T}$ cells isolated from mice with chronic pancreatitis produce large amounts of IL-13. However, it is possible that type 2 innate lymphoid cells (ILC2) stimulated by IL-33 also contribute to the IL-13 production, but this seems unlikely in view of the fact that Rag1-deficient mice do not develop IL-13-medaited fibrosis in the chronic pancreatitis model. ${ }^{67}$ Xue et al. ${ }^{111}$ have suggested that pancreatic stellate cells are a source of IL-13, but this is again unlikely, since staining studies indicate that cells with stellate cell markers are not congruent with cells producing IL-13. ${ }^{67}$ As noted above, IL-13 induction of M2-macrophages may be an important mechanism of IL-13 induction of fibrosis inasmuch as these cells produce TGF- $\beta 1$ and other factors that have direct effects on fibrosis induction and indirect effects via stimulation of pancreatic stellate cells. The latter is supported by the fact that neutralization of IL-13 in the chronic pancreatitis model reduces the number of stellate cells. ${ }^{67,111}$

Recent studies by Ochi et al. ${ }^{125}$ have delineated TLR4 signaling pathways that favor APC induction of chronic 
pancreatitis and/or pancreatitis-associated pancreatic cancer. They have provided evidence that dendritic cells responding to pancreatic antigens support Th2 responses in the absence of the myeloid differentiation primary response gene 88 (MyD88) signaling and that these cells support both pancreatitis and pancreatitis-associated cancer development via TIR-domaincontaining adapter-inducing interferon- $\beta$ (TRIF) signaling. It remains to be seen how activation of these alternative signaling pathways relate to various type of cytokine secretion such as IL-33 secretion.

The clinical course of chronic pancreatitis in humans is composed of acute exacerbation phases and stable quiescent phases. $^{2}$ It is likely that the latter (stable) phases are due to immunosuppressive responses mediated by Tregs with the capacity to suppress pathogenic adaptive immune responses. Indeed it has been shown that Tregs are present in human chronic pancreatic lesions and mediate suppressor effects via their production of IL-10. ${ }^{126}$ These findings in humans are supported by studies of murine models of pancreatitis in that the severity of cerulein-induced acute pancreatitis and the production of TNF- $\alpha$ was shown to be increased following neutralization of endogenous IL-10. ${ }^{127}$ In addition, Demols et al. ${ }^{128}$ report that IL-10 deficiency promotes pancreas fibrosis induced by chronic cerulein challenge and that this fibrogenic response is accompanied by elevated expression of intrapancreatic TGF- $\beta 1$. It should be noted, however, that in these mouse studies the cellular source of the IL-10 was not identified and is not yet clear that IL-10 production by regulatory T cells is the only mechanism mediating the quiescent state in chronic pancreatitis.

\section{CONCLUSION}

In this review, we have summarized the complex chain of events that are responsible for the development of acute and chronic pancreatitis. It is now evident that whereas dysregulation of intra-acinar regulation of proteolytic enzymes are the initial spark that ignites the pancreatic inflammatory process, activation of innate immune responses stimulated by translocated commensal organisms is an indispensible element in sustaining and widening the inflammation. Of considerable interest, an unexpected and essential component of that innate response is the stimulation of NOD1 responses in acinar cells and through such stimulation the enhancement of essential pro-inflammatory signaling leading to the activation of NF- $\kappa \mathrm{B}$ or the de novo production of type I IFN. Finally, a somewhat unique feature of pancreatic inflammation, especially chronic inflammation is the release of IL-33 from damaged acinar cells and thereby the initiation of a pro-fibrotic program mediated by T-cell production of IL-13 and other downstream fibrogenic mediators. It is hoped that this new understanding of pancreatitis will lead to innovative new approaches to its treatment.

\section{ACKNOWLEDGMENTS}

This work was supported by Grant-in-Aid for Scientific Research (15K15370) from the Japan Society for the Promotion of Science, the Kato Memorial Trust for Nambyo Research, and the Health and Labor
Sciences Research Grants for Research on Intractable Diseases from the Ministry of Health, Labor and Welfare, Japan.

\section{DISCLOSURE}

The authors declared no conflict of interest.

(c) 2017 Society for Mucosal Immunology

\section{REFERENCES}

1. Frossard, J.L., Steer, M.L. \& Pastor, C.M. Acute pancreatitis. Lancet $\mathbf{3 7 1}$, 143-152 (2008).

2. Braganza, J.M., Lee, S.H., McCloy, R.F. \& McMahon, M.J. Chronic pancreatitis. Lancet 377, 1184-1197 (2011).

3. Steer, M.L., Waxman, I. \& Freedman, S. Chronic pancreatitis. N. Engl. J. Med. 332, 1482-1490 (1995).

4. Steinberg, W. \& Tenner, S. Acute pancreatitis. N. Engl. J. Med. 330, 1198 1210 (1994).

5. Lankisch, P.G., Apte, M. \& Banks, P.A. Acute pancreatitis. Lancet 386, 85-96 (2015).

6. Logsdon, C.D. \& Ji, B. The role of protein synthesis and digestive enzymes in acinar cell injury. Nat. Rev. Gastroenterol. Hepatol. 10, 362-370 (2013).

7. Cosen-Binker, L.I. \& Gaisano, H.Y. Recent insights into the cellular mechanisms of acute pancreatitis. Can. J. Gastroenterol. 21, 19-24 (2007).

8. Saluja, A.K., Lerch, M.M., Phillips, P.A. \& Dudeja, V. Why does pancreatic overstimulation cause pancreatitis?. Annu. Rev. Physiol. 69, 249-269 (2007).

9. Bhatia, M. et al. Pathophysiology of acute pancreatitis. Pancreatology 5 , 132-144 (2005).

10. Whitcomb, D.C. Genetic aspects of pancreatitis. Annu. Rev. Med. 61, 413-424 (2010).

11. Dawra, R. et al. Intra-acinar trypsinogen activation mediates early stages of pancreatic injury but not inflammation in mice with acute pancreatitis. Gastroenterology 141, 2210-2217. e2212 (2011).

12. Sah, R.P., Dudeja, V., Dawra, R.K. \& Saluja, A.K. Cerulein-induced chronic pancreatitis does not require intra-acinar activation of trypsinogen in mice. Gastroenterology 144, 1076-1085. e1072 (2013).

13. Nemeth, B.C., Wartmann, T., Halangk, W. \& Sahin-Toth, M. Autoactivation of mouse trypsinogens is regulated by chymotrypsin $C$ via cleavage of the autolysis loop. J. Biol. Chem. 288, 24049-24062 (2013).

14. Gaiser, S. et al. Intracellular activation of trypsinogen in transgenic mice induces acute but not chronic pancreatitis. Gut 60, 1379-1388 (2011).

15. Ji, B. \& Logsdon, C.D. Digesting new information about the role of trypsin in pancreatitis. Gastroenterology 141, 1972-1975 (2011).

16. Hoque, R. \& Mehal, W.Z. Inflammasomes in pancreatic physiology and disease. Am. J. Physiol. Gastrointest. Liver Physiol. 308, G643-G651 (2015).

17. Fukui, H., Brauner, B., Bode, J.C. \& Bode, C. Plasma endotoxin concentrations in patients with alcoholic and non-alcoholic liver disease: reevaluation with an improved chromogenic assay. J. Hepatol. 12, 162-169 (1991).

18. Satoh, A., Gukovskaya, A.S., Reeve, J.R. Jr., Shimosegawa, T. \& Pandol, S.J. Ethanol sensitizes NF-kappaB activation in pancreatic acinar cells through effects on protein kinase C-epsilon. Am. J. Physiol. Gastrointest. Liver Physiol. 291, G432-G438 (2006).

19. Alexandre, M., Pandol, S.J., Gorelick, F.S. \& Thrower, E.C. The emerging role of smoking in the development of pancreatitis. Pancreatology 11, 469-474 (2011).

20. Mizushima, N., Levine, B., Cuervo, A.M. \& Klionsky, D.J. Autophagy fights disease through cellular self-digestion. Nature 451, 1069-1075 (2008).

21. Gukovskaya, A.S. \& Gukovsky, I. Autophagy and pancreatitis. Am. J. Physiol. Gastrointest. Liver Physiol. 303, G993-G1003 (2012).

22. Gukovsky, I., Li, N., Todoric, J., Gukovskaya, A. \& Karin, M. Inflammation, autophagy, and obesity: common features in the pathogenesis of pancreatitis and pancreatic cancer. Gastroenterology 144, 1199-1209. e1194 (2013).

23. Diakopoulos, K.N. et al. Impaired autophagy induces chronic atrophic pancreatitis in mice via sex- and nutrition-dependent processes. Gastroenterology 148, 626-638. e617 (2015). 
24. Antonucci, L. et al. Basal autophagy maintains pancreatic acinar cell homeostasis and protein synthesis and prevents ER stress. Proc. Natl Acad. Sci. USA 112, E6166-E6174 (2015).

25. Li, N. et al. Loss of acinar cell IKKalpha triggers spontaneous pancreatitis in mice. J. Clin. Invest. 123, 2231-2243 (2013).

26. Fortunato, F. et al. Impaired autolysosome formation correlates with Lamp-2 depletion: role of apoptosis, autophagy, and necrosis in pancreatitis. Gastroenterology 137, 350-360. 360 e351-355 (2009).

27. Mareninova, O.A. et al. Lysosome associated membrane proteins maintain pancreatic acinar cell homeostasis: LAMP-2 deficient mice develop pancreatitis. Cell. Mol. Gastroenterol. Hepatol. 1, 678-694 (2015).

28. Mareninova, O.A. et al. Impaired autophagic flux mediates acinar cell vacuole formation and trypsinogen activation in rodent models of acute pancreatitis. J. Clin. Invest. 119, 3340-3355 (2009).

29. Mareninova, O.A. et al. Cell death in pancreatitis: caspases protect from necrotizing pancreatitis. J. Biol. Chem. 281, 3370-3381 (2006).

30. Conrad, M., Angeli, J.P., Vandenabeele, P. \& Stockwell, B.R. Regulated necrosis: disease relevance and therapeutic opportunities. Nat. Rev. Drug Discov. 15, 348-366 (2016).

31. Pasparakis, M. \& Vandenabeele, P. Necroptosis and its role in inflammation. Nature 517, 311-320 (2015).

32. He, S. et al. Receptor interacting protein kinase-3 determines cellular necrotic response to TNF-alpha. Cell 137, 1100-1111 (2009).

33. Wu, J. et al. Mlkl knockout mice demonstrate the indispensable role of Mlkl in necroptosis. Cell Res. 23, 994-1006 (2013).

34. Seifert, L. et al. The necrosome promotes pancreatic oncogenesis via CXCL1 and Mincle-induced immune suppression. Nature 532, 245-249 (2016).

35. Kono, H. \& Rock, K.L. How dying cells alert the immune system to danger. Nat. Rev. Immunol. 8, 279-289 (2008).

36. Hoque, R., Malik, A.F., Gorelick, F. \& Mehal, W.Z. Sterile inflammatory response in acute pancreatitis. Pancreas 41, 353-357 (2012).

37. Kang, R. et al. Intracellular Hmgb1 inhibits inflammatory nucleosome release and limits acute pancreatitis in mice. Gastroenterology 146, 1097-1107 (2014).

38. Yasuda, T. et al. Increase of high-mobility group box chromosomal protein 1 in blood and injured organs in experimental severe acute pancreatitis. Pancreas 34, 487-488 (2007).

39. Yasuda, T. et al. Significant increase of serum high-mobility group box chromosomal protein 1 levels in patients with severe acute pancreatitis. Pancreas 33, 359-363 (2006).

40. Sawa, H. et al. Blockade of high mobility group box-1 protein attenuates experimental severe acute pancreatitis. World J. Gastroenterol. 12, 7666-7670 (2006).

41. Hoque, R. et al. TLR9 and the NLRP3 inflammasome link acinar cell death with inflammation in acute pancreatitis. Gastroenterology 141, 358-369 (2011).

42. Akira, S. \& Takeda, K. Toll-like receptor signalling. Nat. Rev. Immunol. 4, 499-511 (2004).

43. Kang, R. et al. The receptor for advanced glycation end products activates the AIM2 inflammasome in acute pancreatitis. J Immunol 196, 4331-4337 ((2016).

44. Kubes, P. \& Mehal, W.Z. Sterile inflammation in the liver. Gastroenterology 143, 1158-1172 (2012).

45. Abdulla, A., Awla, D., Thorlacius, H. \& Regner, S. Role of neutrophils in the activation of trypsinogen in severe acute pancreatitis. J. Leukoc. Biol. 90, 975-982 (2011).

46. Frossard, J.L. et al. The role of intercellular adhesion molecule 1 and neutrophils in acute pancreatitis and pancreatitis-associated lung injury. Gastroenterology 116, 694-701 (1999).

47. Merza, M. et al. Neutrophil extracellular traps induce trypsin activation, inflammation, and tissue damage in mice with severe acute pancreatitis. Gastroenterology 149, 1920-1931. e1928 (2015).

48. Lande, R. et al. Neutrophils activate plasmacytoid dendritic cells by releasing self-DNA-peptide complexes in systemic lupus erythematosus. Sci. Transl. Med. 3, 73ra19 (2011).

49. Garcia-Romo, G.S. et al. Netting neutrophils are major inducers of type I IFN production in pediatric systemic lupus erythematosus. Sci. Transl. Med. 3, 73ra20 (2011).
50. Bedrosian, A.S. et al. Dendritic cells promote pancreatic viability in mice with acute pancreatitis. Gastroenterology 141, 1915-1926. e1911-1914 (2011).

51. Foitzik, T. The enteral factor in pancreatic infection. Pancreatology $\mathbf{1}$, 217-223 (2001).

52. Flint, R.S. \& Windsor, J.A. The role of the intestine in the pathophysiology and management of severe acute pancreatitis. HPB (Oxford) 5, 69-85 (2003).

53. Li, Q., Wang, C., Tang, C., He, Q., Li, N. \& Li, J. Bacteremia in patients with acute pancreatitis as revealed by $16 \mathrm{~S}$ ribosomal RNA gene-based techniques*. Crit. Care Med. 41, 1938-1950 (2013).

54. Runkel, N.S., Moody, F.G., Smith, G.S., Rodriguez, L.F., LaRocco, M.T. \& Miller, T.A. The role of the gut in the development of sepsis in acute pancreatitis. J. Surg. Res. 51, 18-23 (1991).

55. Gianotti, L., Munda, R., Alexander, J.W., Tchervenkov, J.I. \& Babcock, G.F. Bacterial translocation: a potential source for infection in acute pancreatitis. Pancreas 8, 551-558 (1993).

56. Medich, D.S., Lee, T.K., Melhem, M.F., Rowe, M.I., Schraut, W.H. \& Lee, K.K. Pathogenesis of pancreatic sepsis. Am. J. Surg. 165, 46-50. discussion 51-42 (1993).

57. Rychter, J.W. et al. Pretreatment but not treatment with probiotics abolishes mouse intestinal barrier dysfunction in acute pancreatitis. Surgery 145, 157-167 (2009).

58. Tsuji, Y., Watanabe, T., Kudo, M., Arai, H., Strober, W. \& Chiba, T. Sensing of commensal organisms by the intracellular sensor NOD1 mediates experimental pancreatitis. Immunity 37, 326-338 (2012).

59. Mithofer, K., Fernandez-del Castillo, C., Ferraro, M.J., Lewandrowski, K., Rattner, D.W. \& Warshaw, A.L. Antibiotic treatment improves survival in experimental acute necrotizing pancreatitis. Gastroenterology 110, 232-240 (1996).

60. Fritz, S. et al. Prophylactic antibiotic treatment is superior to therapy ondemand in experimental necrotising pancreatitis. Crit. Care 12, R141 (2008).

61. Foitzik, T., Fernandez-del Castillo, C., Ferraro, M.J., Mithofer, K., Rattner, D.W. \& Warshaw, A.L. Pathogenesis and prevention of early pancreatic infection in experimental acute necrotizing pancreatitis. Ann. Surg. 222, 179-185 (1995).

62. Chen, G., Shaw, M.H., Kim, Y.G. \& Nunez, G. NOD-like receptors: role in innate immunity and inflammatory disease. Annu. Rev. Pathol. 4, 365-398 (2009).

63. Strober, W., Murray, P.J., Kitani, A. \& Watanabe, T. Signalling pathways and molecular interactions of NOD1 and NOD2. Nat Rev Immuno/ 6, 9-20 (2006).

64. Sharif, R. et al. Impact of toll-like receptor 4 on the severity of acute pancreatitis and pancreatitis-associated lung injury in mice. Gut $\mathbf{5 8}$, 813-819 (2009).

65. Pastor, C.M. et al. Role of Toll-like receptor 4 on pancreatic and pulmonary injury in a mice model of acute pancreatitis associated with endotoxemia. Crit. Care Med. 32, 1759-1763 (2004).

66. Gao, H.K., Zhou, Z.G., Li, Y. \& Chen, Y.Q. Toll-like receptor 4 Asp299Gly polymorphism is associated with an increased risk of pancreatic necrotic infection in acute pancreatitis: a study in the Chinese population. Pancreas 34, 295-298 (2007).

67. Watanabe, T. et al. Nucleotide-binding oligomerization domain 1 acts in concert with the cholecystokinin receptor agonist, cerulein, to induce IL-33-dependent chronic pancreatitis. Mucosal Immunol. 9, 1234-1249 (2016).

68. Girardin, S.E. et al. Nod1 detects a unique muropeptide from gramnegative bacterial peptidoglycan. Science 300, 1584-1587 (2003).

69. Kim, J.G., Lee, S.J. \& Kagnoff, M.F. Nod1 is an essential signal transducer in intestinal epithelial cells infected with bacteria that avoid recognition by toll-like receptors. Infect. Immun. 72, 1487-1495 (2004).

70. Viala, J. et al. Nod1 responds to peptidoglycan delivered by the Helicobacter pylori cag pathogenicity island. Nat. Immunol. 5, 1166-1174 (2004).

71. Watanabe, T. et al. NOD1 contributes to mouse host defense against Helicobacter pylori via induction of type I IFN and activation of the ISGF3 signaling pathway. J. Clin. Invest. 120, 1645-1662 (2010). 
72. Bouskra, D. et al. Lymphoid tissue genesis induced by commensals through NOD1 regulates intestinal homeostasis. Nature 456, 507-510 (2008).

73. Rakonczay, Z. Jr, Hegyi, P., Takacs, T., McCarroll, J. \& Saluja, A.K. The role of NF-kappaB activation in the pathogenesis of acute pancreatitis. Gut 57, 259-267 (2008).

74. Sah, R.P. \& Saluja, A. Molecular mechanisms of pancreatic injury. Curr. Opin. Gastroenterol. 27, 444-451 (2011).

75. Steinle, A.U., Weidenbach, H., Wagner, M., Adler, G. \& Schmid, R.M. NF-kappaB/Rel activation in cerulein pancreatitis. Gastroenterology $\mathbf{1 1 6}$, 420-430 (1999).

76. Gukovsky, I., Gukovskaya, A.S., Blinman, T.A., Zaninovic, V. \& Pandol, S.J. Early NF-kappaB activation is associated with hormoneinduced pancreatitis. Am. J. Physiol. 275, G1402-G1414 (1998).

77. Gukovskaya, A.S. et al. Pancreatic acinar cells produce, release, and respond to tumor necrosis factor-alpha. Role in regulating cell death and pancreatitis. J. Clin. Invest. 100, 1853-1862 (1997).

78. Grady, T., Liang, P., Ernst, S.A. \& Logsdon, C.D. Chemokine gene expression in rat pancreatic acinar cells is an early event associated with acute pancreatitis. Gastroenterology 113, 1966-1975 (1997).

79. Altavilla, D. et al. Attenuated cerulein-induced pancreatitis in nuclear factor-kappaB-deficient mice. Lab. Invest. 83, 1723-1732 (2003).

80. Ethridge, R.T., Hashimoto, K., Chung, D.H., Ehlers, R.A., Rajaraman, S. \& Evers, B.M. Selective inhibition of NF-kappaB attenuates the severity of cerulein-induced acute pancreatitis. J. Am. Coll. Surg. 195, 497-505 (2002).

81. Letoha, T. et al. In vitro and in vivo nuclear factor-kappaB inhibitory effects of the cell-penetrating penetratin peptide. Mol. Pharmacol. 69, 2027-2036 (2006).

82. Chen, X., Ji, B., Han, B., Ernst, S.A., Simeone, D. \& Logsdon, C.D. NF-kappaB activation in pancreas induces pancreatic and systemic inflammatory response. Gastroenterology 122, 448-457 (2002).

83. Baumann, B. et al. Constitutive IKK2 activation in acinar cells is sufficient to induce pancreatitis in vivo. J. Clin. Invest. 117, 1502-1513 (2007).

84. Satoh, A. et al. PKC-delta and -epsilon regulate NF-kappaB activation induced by cholecystokinin and TNF-alpha in pancreatic acinar cells. Am. J. Physiol. Gastrointest. Liver. Physiol. 287, G582-G591 (2004).

85. Gukovsky, I. et al. Phosphatidylinositide 3-kinase gamma regulates key pathologic responses to cholecystokinin in pancreatic acinar cells. Gastroenterology 126, 554-566 (2004).

86. Tando, Y., Algul, H., Wagner, M., Weidenbach, H., Adler, G. \& Schmid, R.M. Caerulein-induced NF-kappaB/Rel activation requires both $\mathrm{Ca} 2+$ and protein kinase C as messengers. Am. J. Physiol. 277, G678G686 (1999).

87. Ji, B., Gaiser, S., Chen, X., Ernst, S.A. \& Logsdon, C.D. Intracellulartrypsin induces pancreatic acinar cell death but not NF-kappaB activation. J. Biol. Chem. 284, 17488-17498 (2009).

88. Han, B., Ji, B. \& Logsdon, C.D. CCK independently activates intracellular trypsinogen and NF-kappaB in rat pancreatic acinar cells. Am. J. Physiol. Cell Physiol. 280, C465-C472 (2001).

89. Zhao, H.F. et al. Anti-monocyte chemoattractant protein 1 gene therapy attenuates experimental chronic pancreatitis induced by dibutyltin dichloride in rats. Gut 54, 1759-1767 (2005).

90. Deshmane, S.L., Kremlev, S., Amini, S. \& Sawaya, B.E. Monocyte chemoattractant protein-1 (MCP-1): an overview. J. Interferon Cytokine Res. 29, 313-326 (2009).

91. Yu, J.H., Kim, K.H. \& Kim, H. SOCS 3 and PPAR-gamma ligands inhibit the expression of IL-6 and TGF-beta1 by regulating JAK2/STAT3 signaling in pancreas. Int. J. Biochem. Cell Biol. 40, 677-688 (2008).

92. Satoh, A. et al. Nuclear factor kappa B expression in peripheral blood mononuclear cells of patients with acute pancreatitis. Pancreas 26, 350-356 (2003).

93. Oiva, J. et al. Patients with acute pancreatitis complicated by organ failure show highly aberrant monocyte signaling profiles assessed by phosphospecific flow cytometry. Crit. Care Med. 38, 1702-1708 (2010).

94. Oiva, J. et al. Acute pancreatitis with organ dysfunction associates with abnormal blood lymphocyte signaling: controlled laboratory study. Crit. Care 14, R207 (2010).
95. Liew, F.Y., Xu, D., Brint, E.K. \& O'Neill, L.A. Negative regulation of toll-like receptor-mediated immune responses. Nat. Rev. Immunol. 5, 446-458 (2005).

96. Lee, P.Y. et al. Type I interferon modulates monocyte recruitment and maturation in chronic inflammation. Am. J. Pathol. 175, 2023-2033 (2009).

97. Mancuso, G. et al. Type I IFN signaling is crucial for host resistance against different species of pathogenic bacteria. J. Immunol. 178, 3126-3133 (2007).

98. Mayer, J., Rau, B., Gansauge, F. \& Beger, H.G. Inflammatory mediators in human acute pancreatitis: clinical and pathophysiological implications. Gut 47, 546-552 (2000).

99. Malmstrom, M.L. et al. Cytokines and organ failure in acute pancreatitis: inflammatory response in acute pancreatitis. Pancreas 41, 271-277 (2012).

100. Berney, T. et al. Serum profiles of interleukin-6, interleukin-8, and interleukin-10 in patients with severe and mild acute pancreatitis. Pancreas 18, 371-377 (1999).

101. Regner, S., Appelros, S., Hjalmarsson, C., Manjer, J., Sadic, J. \& Borgstrom, A. Monocyte chemoattractant protein 1, active carboxypeptidase $\mathrm{B}$ and CAPAP at hospital admission are predictive markers for severe acute pancreatitis. Pancreatology 8, 42-49 (2008).

102. Sakai, Y., Masamune, A., Satoh, A., Nishihira, J., Yamagiwa, T. \& Shimosegawa, T. Macrophage migration inhibitory factor is a critical mediator of severe acute pancreatitis. Gastroenterology 124, 725-736 (2003).

103. Chao, K.C., Chao, K.F., Chuang, C.C. \& Liu, S.H. Blockade of interleukin 6 accelerates acinar cell apoptosis and attenuates experimental acute pancreatitis in vivo. Br. J. Surg. 93, 332-338 (2006).

104. Cuzzocrea, S. et al. Absence of endogenous interleukin-6 enhances the inflammatory response during acute pancreatitis induced by cerulein in mice. Cytokine 18, 274-285 (2002).

105. Sendler, M. et al. Tumour necrosis factor alpha secretion induces protease activation and acinar cell necrosis in acute experimental pancreatitis in mice. Gut 62, 430-439 (2013).

106. Oruc, N. et al. Infliximab: a new therapeutic agent in acute pancreatitis? Pancreas 28, e1-e8 (2004).

107. Saeki, K. et al. CCL2-induced migration and SOCS3-mediated activation of macrophages are involved in cerulein-induced pancreatitis in mice. Gastroenterology 142, 1010-1020. e1019 (2012).

108. Cayrol, C. \& Girard, J.P. IL-33: an alarmin cytokine with crucial roles in innate immunity, inflammation and allergy. Curr. Opin. Immunol. 31C, 31-37 (2014).

109. Jiang, Y., An, Y., Jiang, D., Wu, B., Yang, Y. \& Sun, D. TNF-alpha regulating interleukin-33 induces acute pancreatic inflammation in rats. Ann. Clin. Lab. Sc.i 46, 54-59 (2016).

110. Kempuraj, D., Twait, E.C., Williard, D.E., Yuan, Z., Meyerholz, D.K. \& Samuel, I. The novel cytokine interleukin-33 activates acinar cell proinflammatory pathways and induces acute pancreatic inflammation in mice. PLoS One 8, e56866 (2013).

111. Xue, J. et al. Alternatively activated macrophages promote pancreatic fibrosis in chronic pancreatitis. Nat. Commun. 6, 7158 (2015).

112. Masamune, A. \& Shimosegawa, T. Pancreatic stellate cells-multifunctional cells in the pancreas. Pancreatology 13, 102-105 (2013).

113. Ouziel, R. et al. The ST2 pathway is involved in acute pancreatitis: a translational study in humans and mice. Am. J. Pathol. 180, 2330-2339 (2012).

114. Sesti-Costa, R. et al. The IL-33/ST2 pathway controls coxsackievirus B5-induced experimental pancreatitis. J. Immunol. 191, 283-292 (2013).

115. Bouma, G. \& Strober, W. The immunological and genetic basis of inflammatory bowel disease. Nat. Rev. Immunol. 3, 521-533 (2003).

116. Strober, W. \& Fuss, I.J. Proinflammatory cytokines in the pathogenesis of inflammatory bowel diseases. Gastroenterology 140 , 1756-1767 (2011).

117. Bhatnagar, A., Wig, J.D. \& Majumdar, S. Expression of activation, adhesion molecules and intracellular cytokines in acute pancreatitis. Immunol. Lett. 77, 133-141 (2001).

118. Bhatnagar, A., Wig, J.D. \& Majumdar, S. Immunological findings in acute and chronic pancreatitis. ANZ J. Surg. 73, 59-64 (2003). 
119. Pietruczuk, M., Dabrowska, M.I., Wereszczynska-Siemiatkowska, U. \& Dabrowski, A. Alteration of peripheral blood lymphocyte subsets in acute pancreatitis. World J. Gastroenterol. 12, 5344-5351 (2006).

120. Hayashi, T., Ishida, Y., Kimura, A., Iwakura, Y., Mukaida, N. \& Kondo, T. IFN-gamma protects cerulein-induced acute pancreatitis by repressing NF-kappa B activation. J. Immunol. 178, 7385-7394 (2007).

121. Park, $H$. et al. A distinct lineage of CD4 $T$ cells regulates tissue inflammation by producing interleukin 17. Nat. Immunol. 6, 11331141 (2005).

122. Harrington, L.E. et al. Interleukin 17-producing CD4 + effector T cells develop via a lineage distinct from the Thelper type 1 and 2 lineages. Nat. Immunol. 6, 1123-1132 (2005).

123. Dai, S.R., Li, Z. \& Zhang, J.B. Serum interleukin 17 as an early prognostic biomarker of severe acute pancreatitis receiving continuous blood purification. Int. J. Artif. Organs 38, 192-198 (2015).
124. Yang, Z.W., Weng, C.Z., Wang, J. \& Xu, P. The role of Card9 overexpression in peripheral blood mononuclear cells from patients with aseptic acute pancreatitis. J. Cell Mol. Med. 20, 441-449 (2016).

125. Ochi, A., Nguyen, A.H., Bedrosian, A.S., Mushlin, H.M. \& Zarbakhsh, S. Barilla Retal. MyD88 inhibition amplifies dendritic cell capacity to promote pancreatic carcinogenesis via Th2 cells. J. Exp. Med. 209, 1671-1687 (2012).

126. Schmitz-Winnenthal, H. et al. Chronic pancreatitis is associated with disease-specific regulatory T-cell responses. Gastroenterology 138, 1178-1188 (2010).

127. Van Laethem, J.L., Eskinazi, R., Louis, H., Rickaert, F., Robberecht, P. \& Deviere, J. Multisystemic production of interleukin 10 limits the severity of acute pancreatitis in mice. Gut 43, 408-413 (1998).

128. Demols, A. et al. Endogenous interleukin-10 modulates fibrosis and regeneration in experimental chronic pancreatitis. Am. J. Physiol. Gastrointest. Liver Physiol. 282, G1105-G1112 (2002). 